\title{
1 Cancer immunotherapy by NC410, a LAIR-2 Fc protein blocking LAIR-collagen interaction
}

2

3 M. Inês Pascoal Ramos ${ }^{1,2}+$, Linjie Tian ${ }^{3}+$, Emma J. de Ruiter ${ }^{4}$, Chang Song ${ }^{3}$, Ana Paucarmayta ${ }^{3}$, Akashdip

4 Singh ${ }^{1,2}$, Eline Elshof ${ }^{1,2}$, Saskia V. Vijver ${ }^{1,2}$, Jahangheer Shaik ${ }^{3}$, Jason Bosiacki ${ }^{3}$, Zachary Cusumano ${ }^{3}$, Linda

5 Liu $^{3}$, Sol Langermann ${ }^{3}$, Stefan Willems ${ }^{4 \&}$, Dallas Flies ${ }^{3 \#}$, Linde Meyaard $^{1,2 \#}$

71 Center for Translational Immunology, University Medical Center Utrecht, Utrecht University, Utrecht,

8 The Netherlands

92 Oncode Institute, Utrecht, The Netherlands

103 NextCure, Beltsville, MD, USA

114 Department of Pathology, University Medical Center Utrecht, Utrecht University, Utrecht, The

12 Netherlands

13

$14+$ these authors contributed equally

15 \# these authors contributed equally

16

17 \&Current affiliation: Department of Pathology and Medical Biology, University Medical Center

18 Groningen, Groningen, The Netherlands

19

20

Correspondence: Linde Meyaard, I.meyaard@umcutrecht.nl and Dallas Flies, fliesd@nextcure.com 


\section{Abstract}

22 Collagens are a primary component of the extracellular matrix and are functional ligands for the inhibitory

23 immune receptor leukocyte associated immunoglobulin-like receptor-1 (LAIR-1). Leukocyte associated

24 immunoglobulin-like receptor-2 (LAIR-2) is a secreted protein that can act as a decoy receptor by binding

25 collagen with higher affinity than LAIR-1. We propose that collagens promote immune evasion by

26 interacting with LAIR-1 and that LAIR-2 could release LAIR-1 mediated immune suppression. Analysis of

27 public datasets shows high LAIR-2 expression being associated with a favorable outcome in certain

28 tumors. We designed a dimeric LAIR-2 with a functional IgG1 Fc tail, NC410, and showed that NC410

29 reduces tumor growth and increases T cell expansion and effector function in humanized tumor models.

30 Immunohistochemical analysis of human tumors shows that NC410 binds to collagen-rich areas where

31 LAIR-1 ${ }^{+}$immune cells are localized. Our findings show that NC410 might be a powerful new strategy for

32 cancer immunotherapy for immune-excluded tumors.

33

34

35

36

37

38

39

40 


\section{Introduction}

42 The introduction of immune checkpoint blockade therapies in the clinic has increased cancer treatment

43 options for a wide range of tumors leading to unprecedented and long-lasting clinical responses. However,

44 not all patients show the same degree of response and not all tumors respond to these therapies $(1,2)$.

45 Thus, identifying novel checkpoints and developing ideal combinations of immunotherapies is essential to

46 optimize and enhance the efficacy of treatment, achieving durable anti-cancer effects with reduced side

47 effects.

48 The extracellular matrix (ECM) is a major structural component in all tissues. It comprises a non-cellular

49 meshwork of proteins, glycoproteins, proteoglycans and polysaccharides with collagens as the most

50 abundant protein. Ongoing ECM remodeling ensures tissue integrity and function, with collagens being

51 synthesized and degraded in a highly regulated manner (3). At least 28 different collagens comprised of

52 at least 43 genes have been identified from which 4 have transmembrane domains allowing expression

53 on the cell surface (4). The ECM functions not only as a scaffold for tissue organization but also provides

54 critical biochemical and biomechanical cues that instruct cell growth, survival, differentiation and

55 migration and regulate vascular development and immune function (5).

56 Several epithelial cancers including breast, pancreatic, colorectal, ovarian, and lung cancer are

57 characterized by a dense ECM where high collagen content correlates with poor prognosis (6). Indeed,

58 ECM or "Matrisome" signatures associated with cancer type and stage of disease have been described (7-

59 10). Cancer associated fibroblasts (CAFs) $(11,12)$, macrophages and tumor cells themselves $(7,13,14)$ all

60 contribute to increased collagen production during cancer progression.

61 The capacity of tumors to induce remodeling of collagens in the tumor microenvironment (TME) was

62 primarily thought to create a suitable microenvironment for tumor cell growth. We now consider

63 abnormal collagen production, composition and organization in the ECM-TME a cause of immune 
64 dysfunction, conveying a chronic wound-healing response instead of anti-tumor immune responses

65 necessary for immune surveillance and the eradication of the tumor $(15,16)$. The abnormal ECM also

66 builds physical barriers to exclude immune cells and therapeutic agents from access to tumor cells (17).

67 Furthermore, tumor-associated collagen can interact with the inhibitory collagen receptor leukocyte

68 associated immunoglobulin-like receptor-1 (LAIR-1)(18). Other collagen-domain containing LAIR-1 ligands

69 have also been reported to be present and enriched in the TME (19). LAIR-1 is an immune checkpoint

70 broadly expressed on the cell surface of immune cells (20) that binds to collagen (21) and molecules with

71 collagen like domains $(22,23)$. LAIR-1 ${ }^{+}$cells strongly adhere to collagen (21). Upon triggering, LAIR-1

72 inhibits NK cell (24), T cell $(25-27)$, B cell $(28,29)$, monocyte $(30)$ and DC function $(31,32)$. Thus, besides

73 formation of a tumor niche, tumor-associated collagens can function to promote immune evasion through

74 its interaction with LAIR-1.

75 We sought to develop an immunomedicine that targets the link between tumor ECM abnormalities and T

76 cell dysfunction to resolve immune suppression and improve tumor clearance. We utilized leukocyte

77 associated immunoglobulin-like receptor-2 (LAIR-2), a soluble collagen binding protein that can act as a

78 decoy for LAIR-1 and as such is a natural immune checkpoint inhibitor $(33,34)$. We developed a dimeric

79 LAIR-2 Fc fusion protein, NC410, as a novel immunomedicine to both target tumor ECM and promote T

80 cell activation through blockade of LAIR-1 mediated inhibition.

81 


\section{Results}

Collagen and LAIR-1 are overexpressed in tumors and correlate with poor overall survival while overexpression of the decoy receptor LAIR-2 associates with increased survival collagen mRNA expression in cancer, we performed a meta-analysis of the TCGA database. 28 collagens comprised of 43 genes were assessed as a group for mRNA expression in cancer and normal tissues across several cancer types, such as lung $(37,38)$, colorectal $(10,39)$ and ovarian cancer $(40,41)$. When we examined the mRNA expression of all collagens genes combined, 13 out of 21 tumors, namely breast invasive carcinoma (BRCA), cholangiocarcinoma ( $\mathrm{CHOL})$, colon adenocarcinoma (COAD), esophageal (ESCA), glioblastoma (GBM), head and neck squamous cell carcinoma (HNSC), kidney renal clear cell carcinoma (KIRC), liver hepatocellular carcinoma (LIHC), lung adenocarcinoma (LUAD), lung squamous cell carcinoma (LUSC), rectum adenocarcinoma (READ), stomach adenocarcinoma (STAD) and thyroid carcinoma (THCA) showed increased collagen mRNA expression compared to healthy tissue (Figure $1 \mathrm{~A}$ top graph). Importantly, this mRNA collagen signature correlated with worse overall survival in 9 out of (SARC), pancreatic (PAAD), esophageal (ESCA), lung (LUAD) and prostate (PRAD) tumors as well as in acute myeloid leukemia (LAML) (Figure 1B). Thus, increased collagen expression and poor clinical outcome associated with overexpression of collagens in multiple cancer types provides a preliminary rationale for targeting collagens as a therapeutic strategy. 
human cancer types when compared to matched healthy tissues or non-tumoral adjacent healthy tissues

107 (Figure 1A, middle graph). By stratifying patients for high and low LAIR-1 mRNA expression we observed that patients with high LAIR-1 mRNA expression had lower survival probability in 4 out of 21 cancers, namely LGG, ESCA, LUAD and LUSC (Figure 1C). We also observed that LAIR-2 mRNA expression, despite being at lower levels than collagen and LAIR-1, was significantly upregulated in 14 out 21 tumors compared to healthy tissue (Figure $1 \mathrm{~A}$ bottom graph). We posited that increased mRNA expression of

112 LAIR-2, the soluble decoy for the LAIR-1 inhibitory receptor, may be associated with improved overall 113 survival. When patients were stratified by high and low LAIR-2 mRNA expression, high expression of LAIR1142 mRNA was indeed associated with increased overall survival probability in 6 out of 30 cancers analysed, namely HNSC, STAD, thyroid carcinoma (THCA), thymoma (THYM), skin cutaneous melanoma (SKCM) and breast invasive carcinoma (BRCA) (Figure 1D). These data support the development of a therapeutic 117 intervention that can inhibit LAIR-1 checkpoint receptor interaction with collagen in the tumor microenvironment of solid cancers.

\section{NC410, a LAIR-2 human IgG1 fusion protein blocks collagen interaction with LAIR-1}

121 Taking advantage of a natural decoy system that would both target cancers and reverse immune 122 inhibition, we generated a LAIR-2 human IgG1 Fc fusion protein for therapeutic use named NC410 (Figure

123 2A). This fusion protein exists as a dimeric protein due to the cysteine bonding in the Fc portion of the 124 protein. NC410 binds to collagen I and III with high avidity by Octet based binding studies and is cross125 reactive to multiple species including rat and mouse due to the conserved nature of collagens across 126 species (Figure 2B). Human LAIR-2 binds with much higher affinity to collagens than human LAIR-1, as 127 reported elsewhere (42). NC410 completely blocks human LAIR-1 binding to collagen I, supporting the 128 high avidity interaction of NC410 and its role as a LAIR-1 decoy therapeutic (Figure 2C). We also 
determined whether NC410 prevents LAIR-1 mediated signaling (Figure 2D, 2E). To do so, we used a reporter cell line that expresses the human LAIR-1 extracellular domain (ECD) fused to CD3z, thus conferring positive signaling capacity to LAIR-1 upon interaction with collagen ligands, and an NFAT-GFP reporter to visualize LAIR-1 mediated signal induction (21). Using flow cytometry (Figure 2D) and Incucyte microscope imaging (Figure 2E and Sup. Figure 1), we observed a dose-dependent inhibition of LAIR-1 NFAT-GFP reporter activity by NC410, indicating that NC410 inhibits collagen-mediated LAIR-1 signaling in a dose-dependent fashion.

\section{NC410 promotes $T$ cell expansion and anti-tumor activity in humanized tumor models}

To study the effect of NC410 on T cell function in vivo, we adoptively transferred human PBMCs into NSG mice. In this model, human xeno-reactive T cells against mouse antigens expand and cause xenogeneicgraft versus-host disease (xeno-GVHD). This model is often used to assess if targeting T cell stimulatory or inhibitory pathways alters xenogeneic T cell activity in vivo. We adapted this model by including the mouse

142 tumor cell line P815 (Figure 3A). In this model, human PBMCs were injected intravenously on day 0, and

143 P815 cells were injected subcutaneously a day later. NC410 or control Fc protein was administered on 144 days 1 and 3 (Figure 3A). NC410 promoted the expansion of human CD8 ${ }^{+}$T cells on day 13, but not on day 1456 in this model (Figure 3B). This was accompanied by reduced tumor growth of P815 after day 14 (Figure $1463 C)$.

147 To determine if the $\mathrm{T}$ cell promoting and anti-tumor effects observed in the xenogeneic P815 model 148 correlated with the capacity of NC410 to elicit T cell anti-tumor activity against an allogeneic human 149 tumor, we developed a humanized subcutaneous tumor model using the HT-29 colorectal cancer cell line, 150 which expresses collagens (Sup. Figure 2). In this model, NSG mice are injected intravenously with human 151 PBMCs, and one day later subcutaneously with HT-29 cells (Figure 3C). NC410 or control treatments began 
on the same day as tumor implantation. NC410 treatment at $10 \mathrm{mg} / \mathrm{kg}$ significantly reduced tumor growth

153 compared to isotype control (Figure 3E). After titration of NC410, tumor volume was reduced in a dose

154 dependent manner (Figure 3F). To investigate whether binding of NC410 to HT29 cells elicits antibody-

155 dependent cellular cytotoxicity (ADCC), we performed in vitro cytotoxicity assays with HT-29 cells, using

156 human PBMC as source of effector cells. We confirmed that HT-29 single cells expressed collagen and that

157 NC410 was able to bind these cells after the EDTA treatment necessary to prepare the cells for the

158 chromium release assay (Sup. Figure 3). In a short-term in vitro chromium release cytotoxicity assay, no

159 differences in HT-29 killing by PBMC were observed between NC410, isotype treated samples and medium

160 (Figure 3G) suggesting that enhancement of ADCC by NC410 is not contributing to tumor reduction in vivo.

161 Thus, we established that NC410 has the capacity to induce T cell expansion and reduction of tumor

162 growth in different in vivo tumor models.

\section{NC410 enhances anti-tumor $T$ cell responses}

Cytokines and chemokines mediate the host-response to cancer by activating and directing the trafficking of immune cells into the TME $(43,44)$. To determine if NC410 promoted infiltration and localized activity

167 of T cells in the TME, immune profiling of HT-29 tumors and spleen tissues was performed at day 27 after

168 treatment (10 mg/kg dose). HT-29 tumors were removed from euthanized mice, weighed, and dissociated

169 for analysis of $\mathrm{T}$ cells and soluble factors. For equal comparison of systemic response, the spleen was

170 harvested by identical means for analysis. Analysis of T cell numbers within the tumor showed a significant

171 increase in the number of $\mathrm{CD}^{+}$and a trend of increased $\mathrm{CD} 8^{+} \mathrm{T}$ cells in tumors treated with NC410

172 compared to isotype control (Figure $4 \mathrm{~A}$ and $\mathrm{B}$ ). To determine the effector capacity of tumor infiltrating $\mathrm{T}$

173 cells (TILs) from NC410-treated mice, we re-stimulated TILs from digested tumors with anti-CD3+anti-

174 CD28 for 5 hours and performed intracellular staining to examine IFN- $\gamma$ and TNF- $\alpha$ production. After re- 
175 stimulation a significant increase in IFN- $\gamma^{+}$and IFN- $\gamma^{+}$TNF- $\alpha^{+}$double positive cells was observed in the

176 NC410 treatment group compared to control. (Figure 4C). Based on this observation, we further assessed

177 cytokines and chemokines in the local TME vs peripheral spleen on day 27 (Figure 4D-E and Sup figure 5).

178 Analysis of cytokines indicated that IFN- $\gamma$ and granzyme B were significantly increased in the TME, but not

179 soluble CD40L (Figure 4D). Increased expression of CD40L and granzyme B was observed in the spleen

180 (Figure 4D). Chemokine analysis indicated that CXCL10, 11 and 12 were all significantly increased in the

181 TME, and significantly decreased in the spleen (Figure 4E). Importantly, the concentration of all three

182 chemokines correlated with tumor reduction (Sup. Figure 4). These results support a role for NC410 in

183 enhancing the recruitment of T cells and liberating their effector function in the TME.

\section{NC410 promotes collagen remodeling}

186 Remodeling of ECM is pivotal to the development and progression of cancer. Recently it has been

187 proposed that specific collagen-derived biomarkers reflecting the turnover of collagens may be used as

tools to non-invasively interrogate cell reactivity in the TME and predict response to treatment (45-47).

189 Because NC410 both engages collagens and promotes local T cell and immune responses, it was surmised

190 that NC410 treatment may result in collagen remodeling. Therefore, we determined whether NC410

191 treatment increased collagen degradation products (CDPs) in the serum of HT-29 tumor bearing mice

192 injected with PBMC over the course of tumor growth and rejection mediated by NC410 (Figure 5A and B).

193 Nine CDPs were analyzed prior to experiment initiation and during four weeks of tumor growth (Figure

194 5C). While there was no change in most CDP, it was interesting that the concentration of two CDPs

195 significantly increased in the serum of NC410 treated mice in comparison to controls. C6M, a collagen VI

196 MMP-2 CDP, and C4GzB, a collagen IV Granzyme B CDP were significantly increased at week 4 (Figure 5D)

197 in the NC410 treated group in comparison to the control group. Interestingly, this increase in serum CDPS 
was observed at the time of NC410 mediated tumor eradication suggesting that the CDPs were derived from the tumor and that the increase in CDPs was a result of T cells activation and effector function.

\section{NC410 binds collagen rich tumors with an immune excluded phenotype}

202

In order to identify tumor types and/or patient groups that would benefit from this therapy, we performed immunohistochemical analysis of serial tissue sections from seven different tumor types, of which we analyzed biopsies from ten patients per tumor type. Sections of head and neck squamous cell carcinoma (HNSC), glioblastoma (GBM), melanoma, non-small-cell lung carcinoma (NSCLC), high-grade serous carcinoma (HGSC), pancreatic ductal adenocarcinoma (PDAC), and stomach adenocarcinoma (STAD), were stained for hematoxylin and eosin, Masson's Trichome, anti-LAIR-1, NC410 and the immune cell markers, CD45, CD3, CD68 and CD163 (Figure 6A). NC410 binding co-localized with Masson's Trichrome positive collagen areas within the TME and LAIR-1 ${ }^{+}$immune cells were present in all tumors (Figure 6B, Sup. Figure 5, heathy tissue Sup. Figure 6). Both myeloid and lymphoid cells within the TME expressed LAIR-1 (Sup. Figure 7). NC410 binding was highest in pancreatic cancer in agreement with a collagen-rich microenvironment (Figure 6C). Head and neck squamous cell carcinoma had the highest number of LAIR$1^{+}$cells (Figure 6C). Importantly, LAIR-1 ${ }^{+}$cells were enriched in NC410 positive areas (Figure 6D) suggesting that LAIR- $1^{+}$cells were entrapped in the collagen matrix. Most human solid tumours exhibit distinct immunological phenotypes being divided into immune inflamed, immune excluded, and immune desert tumors on the basis of immune cell infiltrate and localization $(48,49)$. By characterizing our cohort according to these immunological phenotypes (Figure 6E, Sup. Figure 8), we observed that NC410 binding was particularly increased in immune excluded tumors, namely HNSC, melanoma, NSCLC and STAD. (Figure 6F). Thus, in immune excluded tumors, LAIR-1 ${ }^{+}$cells are sequestered in collagen-rich areas where NC410 can bind. 


\section{Discussion}

222 Cancer cells have evolved multiple mechanisms to escape immune surveillance, such as defects in antigen

223 presentation machinery, the recruitment of immunosuppressive cell populations and the upregulation of

224 negative regulatory pathways, all resulting in hampered effector function of immune cells and the

225 abrogation of antitumor immune responses (50). Tumor progression is also accompanied by extensive

226 remodeling of the extracellular matrix leading to the formation of a tumor-specific ECM, which is often

227 more collagen-rich and of increased stiffness $(51,52)$. Collagen expression and density have been shown

228 to be associated with a worse prognosis, either by directly promoting tumor growth or by preventing

229 immune cell infiltration (8).

We have shown that a combined collagen signature was associated with a worse prognosis in 13 out of

21 tumor types supporting the notion that increased collagen expression is detrimental for overall survival

232 in tumors. Importantly, LAIR-1 mRNA expression was associated with a worse prognosis in 4 out of 21

233 tumors and LAIR-2 mRNA expression was associated with a better prognosis in 6 out of 21 tumors. This

234 indicates that targeting LAIR-1-collagen interaction might be helpful in a specific group of tumors.

235 It has been demonstrated that collagens can also directly modulate $T$ cell function(53-55), for instance

236 through the inhibitory collagen receptor LAIR-1 $(18,27)$. We hypothesize that increased expression and

237 remodeling of collagen in the TME serves to set a threshold of T cell activation through LAIR-1 and is

238 employed by tumor cells to escape immune surveillance. In this study we show that disrupting collagen

239 interaction with LAIR-1 by means of a dimeric LAIR-2 Fc fusion protein has a therapeutic effect in cancer

240 models. NC410 administration in humanized mouse tumor models results in a reduction of tumor volume

241 by increasing T cell expansion and cytotoxic activity but does not enhance ADCC in vitro. NC410 binds to

242 collagen-rich areas of tumors, where LAIR-1+ cells are sequestered. NC410 also binds to healthy collagen, 
therefore next to the direct effect on T cell mobilization and activation in the tumor, systemic effects could

244 also add to its therapeutic potential.

245 During ECM turnover, proteolytically cleaved matrix degradation fragments are released into the systemic

246 circulation (3). These small protein fragments containing specific protease-generated neo-epitopes, or

247 'protein fingerprints', can be used as serological biomarkers directly reflecting disease pathogenesis.

248 Several studies have shown that serum levels of collagen degradation fragments are elevated in cancer

249 patients compared to healthy controls (56-58), and that checkpoint blockade may alter serum CDP levels

250 (47). We observed that specific fragments generated by degradation of collagen VI by MMP-2 (C6M) and

251 collagen IV by granzyme B (C4GzB) were increased after NC410 treatment. The increase of circulating C6M

252 and $\mathrm{C} 4 \mathrm{GzB}$ coincided with reduction of tumor volume suggesting that these fragments might be generated

253 by increased infiltration and/or activation of T cells in the TME. C6M and C4GzB collagen fragments have

254 the potential to be biomarkers of NC410 response, which will be investigated in a recently initiated NC410

255 first-in-human clinical trial (NCT04408599).

256 In the era of personalized medicine, it is very important to address how to select patients that are likely

257 to benefit from immune checkpoint blockade therapy. Within different tumor types a distinction based

258 on the presence and location of immune infiltrates can be made, separating patients into having immune

259 excluded, immune inflamed and immune desert tumors. Currently, immune inflamed tumors, where the

260 majority of immune cells are present throughout the neoplastic cells, have been associated with better

261 response to currently available checkpoint blockade therapy compared with the other phenotypes (59,

262 60). The immune-excluded phenotype is characterized by the presence of immune cells that cannot

263 penetrate the parenchyma of the tumors but instead are located in the stroma that surrounds the cancer

264 cells (61). These tumors are generally resistant to current checkpoint blockade therapy (62). We

265 performed an immunohistochemical screen across 7 tumor types, analyzing biopsies of 10 individual 
patients per tumor type, and observed that immune excluded tumors had the highest NC410 binding.

267 Importantly we observed that LAIR-1 ${ }^{+}$cells were present within these NC410-binding collagen rich areas.

268 This might indicate that an abundance of collagen keeps the immune cells trapped in the tumor stroma, possibly by binding to LAIR-1. Treatment with NC410 could block this interaction resulting in LAIR-1 $1^{+}$cell activation and infiltration into the tumor nests, promoting tumor clearance.

271 LAIR-2 acts as a decoy receptor by binding to collagen with higher affinity than LAIR-1 and therefore 272 antagonizes LAIR-1 inhibitory function $(33,34)$. Circulating LAIR-2 protein concentration is low or 273 undetectable in healthy individuals $(33,42)$. In the presence of limited endogenous LAIR-2, LAIR-1 can 274 bind collagens, thereby allowing the inhibitory receptor to signal and prevent or reduce T cell activity 275 and/or retain LAIR-1+ cells in collagen-rich areas. In the TME increased levels of collagens, in absence of 276 increased LAIR-2, will therefore promote tumor immune escape. Our analysis of TCGA data indeed 277 revealed that enhanced expression of endogenous LAIR-2 in some cancers associated with better 278 prognosis, suggesting that further increasing LAIR-2 in vivo would have a therapeutic advantage. NC410 279 has a higher avidity to collagen than endogenous LAIR-2 due to its dimeric structure, since in vivo LAIR-2 280 is expressed as a monomer, enhancing its potential to block the inhibitory capacity of membrane-bound 281 LAIR-1. NC410 binds both healthy and tumoral collagen and theoretically could be sequestered before 282 reaching the tumor site. We hypothesize that the avidity of NC410 towards tumoral collagen is higher 283 than to healthy collagen, therefore resulting in a therapeutic effect, but this needs additional study. Other 284 LAIR-1 ligands, such as C1q have been shown to be increased in tumors (19) and may also provide a local 285 inhibitory effect that could be potentially reversed by NC410.

286 Taken together, tumor patients with collagen rich tumors that present an immune excluded phenotype 287 and low endogenous LAIR-2 expression would be predicted to benefit the most from NC410 therapy. Our 288 immunohistochemical analysis points to HNSC, melanoma, NSCLC and STAD as immune excluded tumor 
289 types with the highest NC410 binding. In agreement with our hypothesis that blocking LAIR-1- collagen

290 interaction in these tumors would be beneficial, TCGA analysis showed that increased LAIR-2 mRNA at the

291 tumor site also increases survival probability in HNSC, melanoma, and STAD. Together, this may be

292 indicative that patients suffering from these particular tumors might benefit the most from NC410

293 treatment.

294 While conducting our study, an unrelated research group generated a structurally similar LAIR-2 Fc fusion

295 protein for use as an immune therapy for cancer to block LAIR-1 inhibitory function (63). Xu et al. showed

296 that LAIR-2 Fc reversed T cell inhibition in vitro and in vivo and promoted anti-tumor immunity in vivo. Our

297 models are non-overlapping with the models described in that study, independently supporting the

298 concept of LAIR-2 Fc for cancer immunotherapy. In addition, we now provide an immunohistochemical

299 rationale for LAIR-2 Fc treatment in certain tumor types by extensive analysis of human tumor samples,

300 as well as potential biomarkers for patient selection and response to therapy.

301 Our data support NC410 as a novel immunomedicine for targeting immune excluded collagen-rich tumors

302 and enabling normalization of the tumor-immune microenvironment. First-in-human studies have 303 recently been initiated with NC410 (NCT04408599). 


\section{Material and Methods}

314 Mice

315 NSG (NOD-SCID IL2Rynull) female mice were purchased at the age of 6 - 8 weeks old from Jackson Labs.

316 Upon arrival at NextCure, mice were divided into 5 - 6 mice per cage and kept in the quarantine room for

317 at least 6 days to acclimate to the environment. All mouse studies were performed at NextCure based on

318 Institutional Animal Care and Use Committee standards.

\section{Bioinformatics}

321 For collagen expression, the mRNA expression of 44 collagen genes in normal and tumor tissue for each

322 cancer type were queried using TCGA database (https://cancergenome.nih.gov/). mRNA collagen data

323 was available in TCGA except for COL6A5. The transcript per million (TPM) values were log2 transformed

324 and averaged per individual across all collagen proteins. The distribution of average expression across

325 individuals was plotted using ggplot utility version 2.3.3.2 in R version 4.0.2. The distribution of expression

326 in normal tissues was compared against those in cancer tissue using a non-parametric Wilcoxon test in R.

327 For overall survival analysis, the log2 transformed collagen expression for each of the 43 collagen genes

328 in various cancers was divided into four quantiles. The patients in lower quantile were considered

329 individuals with low expression and those in the upper quantile were considered those with high

330 expression. The estimate of survival based on collagen expression in the tumor was determined using the

331 Kaplan-Meier method. The survival curves were drawn using ggsurvplot function in the survminer $\mathrm{R}$

332 package. The survival curves for the protein-cancer combination where the $p$-value was less than 0.05 
were considered significant. The expression and overall survival analyses for LAIR-1 and LAIR-2 were performed in the same manner. $\mathrm{R}$ codes can be found in the source code files.

For collagen gene expression analysis of HT-29 cells, data was acquired from the public dataset GSE41586

338 plotted using the ggplot package (v3.3.2).

\section{Cells and antibodies}

2B4 T cell hybridoma cells transduced with a NFAT-GFP reporter and hLAIR-1-CD3ろ, the hLAIR-1 reporter cells, or transduced with a NFAT-GFP reporter and CD3ろ, the WT reporter cells (21), were cultured in RPMI 1640 (Life Technologies) supplemented with 10\% Fetal Bovine Serum (FBS) (Sigma-Aldrich) and 1\% penicillin/streptomycin (Gibco). P815 cells (ATCC) were cultured in DMEM, 2 mM L-glutamine, 25 mM HEPES, 10\% FBS and Penicillin-Streptomycin (100 U/mL-100 $\mu \mathrm{g} / \mathrm{mL}$ ). HT-29 cells (ATCC) were cultured in

$347 \mathrm{CHO}$ cells were cultured in CD CHO medium (ThermoFisher). Anti-human CD45 (hCD45)-BV421, anti348 mouse CD45(mCD45)-APC, anti-human CD3(hCD3)-Percp.Cy5.5, anti-human CD8(hCD8)-AF488, anti349 human TNF- $\alpha$ (hTNF- $\alpha$ )-PE and anti-human IFN- $\gamma$ (hIFN- $\gamma$ )-PECy7 were from ThermoFisher. Anti-human CD4 (hCD4)-BV711 was from Biolegend.

\section{LAIR-1-Fc and LAIR-2-Fc (NC410) generation}

353 The human LAIR-1 and LAIR-2 genes were synthesized by GeneArt and genetically fused with the $\mathrm{N}$ 354 terminus of IgG1 Fc domain. A stable CHO cell line expressing recombinant human LAIR-1 Fc or LAIR-2 Fc 
fusion protein was developed using the Lonza GS system. Briefly, $5 \times 10^{7} \mathrm{CHO}$ cells were transfected by electroporation using $80 \mu \mathrm{g}$ of linearized plasmid DNA in a $0.4 \mathrm{~cm}$ cuvette. Following electroporation (300 $\mathrm{V}, 900 \mu \mathrm{F}$ ) cells were resuspended in $100 \mathrm{~mL}$ glutamine-free CD CHO medium (ThermoFisher). The following day, MSX (Millipore) was added to a final concentration of $50 \mu \mathrm{M}$, and cells were monitored for the next two weeks as prototrophic cells began to grow. Single clone of stably transfected cells was cultured, and supernatant was harvested and purified by affinity chromatography. The protein purity was determined by HPLC and sodium dodecyl sulfate-polyacrylamide gel electrophoresis. For the LAIR-1collagen binding assay, LAIR-1 Fc was biotinylated with EZ-Link NHS-PEG4-Biotin (ThermoFisher), NoWeight Format (ThermoFisher) and free biotin was removed by ZebaSpin Desalting Columns

364 (ThermoFisher) following manufacturer's instructions. consent from the subjects in accordance with the Declaration of Helsinki) using standard Ficoll density

370 gradient centrifugation. Briefly, blood was diluted 1:1 with PBS and layered on top of $15 \mathrm{~mL}$ of Ficoll-Paque

371 (GE healthcare) in $50 \mathrm{~mL}$ conical tube. Suspension was centrifuged at $400 \times \mathrm{g}$ for $20 \mathrm{~min}$ at $20^{\circ} \mathrm{C}$ in a

372 swinging bucket rotor without brake. The mononuclear cell layer at the interphase was carefully collected

373 and transferred to a new $50 \mathrm{~mL}$ conical tube. The cells were washed with PBS and centrifuged at $300 \times \mathrm{g}$

374 for $10 \mathrm{~min}$ at $20^{\circ} \mathrm{C}$. The supernatant was discarded, and the cell pellet was washed twice with $50 \mathrm{~mL}$ PBS.

375 The isolated PBMCs were immediately used for in vitro studies. 


\section{Binding and Blocking Studies}

Octet avidity analysis

A ForteBio Octet RED96 instrument was used for avidity assessments. The anti-human Fc antibody capture sensors (ForteBio) were first loaded with LAIR-2-Fc followed by an association step where the loaded sensor was dipped into wells containing human, mouse or rat collagen I (human, R\&D Systems; mouse, protein was diluted in assay buffer (ForteBio) at $20 \mu \mathrm{g} / \mathrm{mL}$ and the collagen concentration ranged from was conducted using the Octet's Data Analysis 9.0 software. with DELFIA Wash buffer (PerkinElmer). The plates were blocked for non-specific binding with 3\% BSA (200 $\mu \mathrm{L} /$ well, Millipore) for 1 hour. Plates were washed 3 times ( $300 \mu \mathrm{L} /$ well) with DELFIA wash buffer and an NC410-biotin and human LAIR-1 Fc mixture (50 $\mu \mathrm{L} /$ well) was added to plates and incubated for 2 hours at ambient temperature. The plates were washed 3 times $(300 \mu \mathrm{L} /$ well) with DELFIA wash buffer.

394 Europium-labeled Streptavidin (Eu-SA) (100 $\mu \mathrm{L} /$ well, PerkinElmer) was diluted 1:1000 in DELFIA assay 395 buffer and was added to plates and incubated for 1 hour at ambient temperature. Following the 396 incubation, the plates were washed with $300 \mu \mathrm{L} /$ well of DELFIA wash buffer. DELFIA enhancement 397 solution was equilibrated to ambient solution during detection antibody incubation. Following the last wash, $100 \mu \mathrm{L}$ of DELFIA enhancement solution (PerkinElmer) was added to each well and incubated on a 
plate shaker for 5 minutes prior to reading on an EnVision plate reader with excitation at $340 \mathrm{~nm}$, and

400

401

402

403

404

405

406

407

408

409

410

411

412

413

414

415

416

417 Plates were prepared similarly to the flow cytometric analysis. After adding reporter cells to collagen and

418 fusion protein treated wells, plates were placed in the Incucyte S3 (Sartorius) and green fluorescence of 419

Flow cytometry blocking studies

To assess the blocking capacity of NC410 a titration assay with LAIR-1 reporter cells was performed as previously described(21). Black Falcon clear flat bottom 96 -well plates were coated with $5 \mu \mathrm{g} / \mathrm{mL}$ human collagen I (Sigma-Aldrich) in 2 mM acetic acid (Merck), anti-mouse-CD3 (BD), anti-human-LAIR-1 antibody (clone 8A8 (21)) in PBS (Sigma-Aldrich) or isotype control (eBiosciences) in PBS by spinning down for three minutes at $1700 \mathrm{rpm}$ and incubating overnight at $4{ }^{\circ} \mathrm{C}$. The next day, plates were washed with PBS and pre-incubated with the indicated concentrations of NC410 or isotype control (Nextcure) in culture medium by spinning down for five minutes at $1500 \mathrm{rpm}$ at room temperature (RT) and incubating for two hours at $37^{\circ} \mathrm{C}$.

WT and hLAIR-1 reporter cells were harvested and seeded at $1 \times 10^{6}$ cells $/ \mathrm{mL}$ in $50 \mu \mathrm{l} /$ well on top of the collagen and fusion proteins treated wells and spun down for three minutes at $1700 \mathrm{rpm}$ at RT. Plates were incubated overnight, approximately 16 hours, at $37{ }^{\circ} \mathrm{C}$ and GFP expression was measured on a LSRFortessa (BD Biosciences).

Incucyte assay the GFP expressed by the reporter cells was imaged every hour for 24 hours. 
420

421

422

423

424

425

426

427

428

429

430

431

432

433

434

435

436

437

438

439

440

441

442

Analysis of Incucyte images was performed using the Incucyte 2020A analysis programme (Sartorius), where green fluorescence was evaluated using Top-Hat segmentation (radius $100 \mu \mathrm{m}$ and threshold 2 $\mathrm{GCU})$, edge split turned on, minimum mean intensity of $3 \mathrm{GCU}$ and an area filter of $600 \mu \mathrm{m}^{2}$ to calculate the total green integrated intensity (GCU $\times \mu \mathrm{m}^{2} /$ Image) per well.

\section{In vivo experiments}

Leukopaks (StemCell) were diluted with PBS and layered with $35 \mathrm{~mL}$ of diluted cell suspension over $15 \mathrm{~mL}$ of Ficoll-Paque (GE healthcare) in $50 \mathrm{~mL}$ conical tube. Suspension was centrifuged at $400 \times \mathrm{g}$ for $30 \mathrm{~min}$ at $20{ }^{\circ} \mathrm{C}$ in a swinging bucket rotor without brake. The mononuclear cell layer at the interphase was carefully collected and transferred to a new $50 \mathrm{~mL}$ conical tube. The cells were washed with PBS and centrifuged at $300 \times \mathrm{g}$ for $10 \mathrm{~min}$ at $20^{\circ} \mathrm{C}$. The supernatant was discarded, and the cell pellet was washed twice with $50 \mathrm{~mL}$ PBS. The isolated PBMCs were frozen and stored in liquid nitrogen.

Prior to in vivo studies, PBMCs were rested overnight in RPMI 1640, 2 mM L-glutamine, 10 mM HEPES, $10 \%$ FBS, Penicillin-Streptomycin $(100 \mathrm{U} / \mathrm{mL}-100 \mu \mathrm{g} / \mathrm{mL}$ ) and $250 \mathrm{U} / \mathrm{mL}$ DNase (Millipore). Female NSG mice were injected intraveneously (i.v.) with $1-2 \times 10^{7}$ PBMC in $100 \mu \mathrm{l}$ of $1 \times$ PBS. The next day, $2 \times 10^{5}$ P815 cells in PBS, or $1 \times 10^{6}$ HT-29 cells in PBS with 50\% Matrigel (Corning) were injected subcutaneously on the right flank. Mice were randomly assigned into treatment or control groups (6 mice per group). The sample size per group was determined with resource equation approach $n=D F / k+1$ where $n=n u m b e r$ of sample per group, $\mathrm{k}=$ number of groups and $\mathrm{DF}=$ degrees of freedom with acceptable range between 10 to 20 in analysis of ANOVA and t test $(64,65)$. Beginning on day 1, LAIR-2 Fc and control protein were injected intraperitoneally (i.p.) Q4D x 4 doses followed by Q7D until the endpoint. Tumor size was monitored 2-3 times a week. Tumor volumes were determined according to the formula tumor volume $=0.5 \times$ (shorter diameter $)^{2} \mathrm{x}$ longer diameter. At endpoint, tumor and spleen tissues were collected for $\mathrm{T}$ cell population 
443

444

445

446

447

448

449

450

451

452

453

454

455

and/or cytokine analysis. In some studies, blood was collected weekly for T cell population and collagen degradation analysis. Mice were randomized in all tumor models prior to treatment. Tumor measurements were performed in a blinded manner.

For ex vivo analysis, cells were stained with Zombie NIR viability dye (Biolegend) in PBS at RT for 10 min. After washing with FACS buffer (2\%FBS in PBS), cells were stained with antibodies against cell surface antigens at $4{ }^{\circ} \mathrm{C}$ for $30 \mathrm{~min}$. For the intracellular staining, cells were stimulated with Cell Stimulation Cocktail plus protein transport inhibitors (ThermoFisher) at $37^{\circ} \mathrm{C}$ for 5 hours followed by Zombie NIR and cell surface antigen staining. After cell fixation and permeabilization, the intracellular TNF- $\alpha$ and IFN- $\gamma$ were stained following the instructions of BD Cytofix/CytoPerm Plus Fixation/Permeabilization Kit. All antibodies were used at the concentrations recommended by manufacturers. Stained cells were washed and resuspended in $150 \mu \mathrm{L}$ FACS buffer and $80 \mu \mathrm{L}$ of samples were acquired on an Attune flow cytometer (ThermoFisher). Human $\mathrm{CD} 4^{+}$and $\mathrm{CD} 8^{+} \mathrm{T}$ cells were gated based on live/ $\mathrm{hCD} 45^{+} \mathrm{mCD} 45^{\mathrm{h} C D} 3^{+} \mathrm{hCD} 4^{+} \mathrm{hCD} 8$ and live $/ \mathrm{hCD}^{+} 5^{+} \mathrm{mCD} 45^{-} \mathrm{hCD} 3^{+} \mathrm{hCD} 4^{-} \mathrm{hCD} 8^{+}$respectively.

To prepare mouse blood T cells for staining, $80-200 \mu \mathrm{L}$ blood was treated with $3 \mathrm{~mL}$ ACK lysis buffer (KD medical) to lyse the red blood cells for 5 min at RT followed by washing with PBS. The initial volume of blood was recorded for calculation of cell counts per $\mathrm{mL}$ of blood according to the formula: Cell counts per $\mathrm{mL}$ of blood= [acquired counts $\times 150 \times 1000] \div$ [initial blood volume $(\mu \mathrm{L}) \times 80]$.

To prepare the single cell population from tumors for staining, tumor tissues were weighed, cut into small pieces, and digested with mouse tumor dissociation kit (Miltenyi) and dissociated with gentleMACS Dissociator (Miltenyi). The tumor weight was recorded for the normalization of cell counts.

To assess cytokines from tissues, tumor and spleen tissues were weighed and cut into small pieces in 1.5mL Eppendorf tube on ice. $200 \mu \mathrm{L}$ of RIPA Lysis buffer (ThermoFisher) was added with proteinase inhibitor (Roche) and $250 \mathrm{U} / \mathrm{mL}$ of DNase (Millipore) and the tissues were dissociated with pellet pestles 
466 (Sigma) on ice. The samples were kept on ice for $30 \mathrm{~min}$, vortexing occasionally. Centrifuge at 10,000 ×g

467 for 20 mins at $4{ }^{\circ} \mathrm{C}$ to pellet cell debris and then transfer the supernatant to a fresh Eppendorf tube without 468 disturbing the pellet. The tissue weight was recorded for the normalization analysis of cytokines.

\section{Luminex cytokine assay}

471 Tissue lysate was used for analysis of the cytokine profile (SDF-1 $\alpha$, IL-2, IL-4, IP-10, IL-10, IL-17A, IFN- $\gamma$,

472 TNF- $\alpha$, I-TAC, granzyme B, sCD40L) using a Luminex assay. The Singleplex Luminex ${ }^{\text {TM }}$ Protein Assay Kit for

473 each cytokine was from ThermoFisher. Antibody-specific capture magnetic beads were added to wells of

474 a 96-well plate. Samples and protein standards were then placed into the microplate wells. After

475 incubation, the beads were washed using a handheld magnet and were resuspended in secondary

476 detection antibody solution followed by washing and addition of streptavidin-RPE. The beads were then

477 washed again for analysis on a FlexMAP 3D ${ }^{\mathrm{TM}}$ (ThermoFisher).

\section{EDTA treatment of HT-29 cells}

480 HT-29 cells were collected from T75 flasks (Thermo Scientific) using different concentrations of EDTA (0.1;

$4810.2 ; 0.5 ; 1$ and $2 \mathrm{mM}$ ) for $10 \mathrm{~min}$ at 37C. Cells were blocked with $10 \%$ BSA/10\% normal mouse serum

482 (NMS)/ 10\%FCS for $15 \mathrm{~min}$ at 4C. Cells were then incubated with biotin labelled NC410 (10ug/ml;

483 Nextcure) in PBS + 1\% BSA buffer for 30 min RT. After washing with PBS + 1\% BSA, by spinning down for 484 five minutes at $1500 \mathrm{rpm}$ at RT, cells were incubated with streptavidin APC (eBioscience) diluted in PBS + $4851 \%$ BSA buffer for 20 min at 4C. Cells were washed again and measured on a FACSCanto (BD biosciences). 


\section{Collagenase treatment of HT-29 cells}

489 HT-29 cells were collected from T75 flasks (Thermo Scientific) using 0.1mM EDTA for 10 min at 37C. Cells

490 were washed and treated with 40 U Collagenase (from Clostridium histolyticum; Sigma) for different time

491 points $(5,10,15,20$ and $30 \mathrm{~min})$ at $37 \mathrm{C}$. Cells were washed, by spinning down for five minutes at 1500

492 rpm at RT, and blocked with 10\% BSA/10\% normal mouse serum (NMS)/ 10\%FCS for 15 min at 4C. Cells

493 were washed and incubated with biotin labelled NC410 (10ug/ml; Nextcure) in PBS $+1 \%$ BSA buffer for

49430 min RT. After washing with PBS + 1\% BSA, by spinning down for five minutes at 1500 rpm at RT, cells

495 were incubated with streptavidin APC (eBioscience) diluted in PBS $+1 \%$ BSA buffer for 20 min at 4C. Cells

496 were washed again and measured on a FACSCanto (BD biosciences).

497

\section{Antibody dependent cellular cytotoxicity (ADCC) Assay}

ADCC with ${ }^{51} \mathrm{Cr}$-labeled target cells was described previously (66). Briefly, HT-29 target cells were labeled

with $100 \mu \mathrm{Ci}(3.7 \mathrm{MBq}){ }^{51} \mathrm{Cr}$ for $3 \mathrm{~h}$ in complete medium. After extensive washing, cells were adjusted to

$10^{5} / \mathrm{mL}$. HT-29 cells were then incubated with NC410 or isotype control for $30 \mathrm{~min}$. Different effector to 


\section{Immunofluorescence staining}

5135000 HT-29 cells were seeded in black Falcon clear flat bottom 96-well plates and cultured for 3 days. Cells

514 were fixed with $4 \%$ paraformaldehyde for $15 \mathrm{~min}$ at room temperature, and blocked with $5 \%$ BSA in PBS

515 for $1 \mathrm{~h}$ at room temperature. Cells were then incubated with isotype control (Nextcure), biotin labelled $516 \mathrm{NC410}$ (10ug/ml, Nextcure) or pan-collagen antibody (Thermo fisher) diluted in PBS $+1 \%$ BSA buffer for 1

$517 \mathrm{~h}$ at room temperature. After thoroughly washing with PBS, the slides were incubated with anti-human

518 IgG1-AlexaFluor 594 or streptavidin Alexa Fluor 594 (Life Technologies- Thermo Fisher Scientific) diluted

519 in PBS + 1\% BSA buffer for $30 \mathrm{~min}$ at room temperature. Slides were finally washed and mounted with

520 DAPI VectaShield hardset (Vector Lab) and allowed to settle before image acquisition on a Zeiss

521 fluorescence microscopy (Zeiss) using the Axiovision software (Zeiss).

\section{Collagen degradation peptide analysis}

524 The mouse serum samples were assessed for collagen degraded peptides (PRO-C3, C3M, C4M, G4GzB,

525 RRO-C6, VICM, CRPM, reCIM) by Nordic Bioscience as previously described $(47,67)$.

\section{Tumor specimens for immunohistochemistry}

528 Specimens of seven selected tumor types were included for analysis: head and neck squamous cell 529 carcinoma (HNSC), glioblastoma (GBM), melanoma, non-small-cell lung carcinoma (NSCLC), high-grade

530 serous carcinoma (HGSC), pancreatic ductal adenocarcinoma (PDAC), and stomach adenocarcinoma 531 (STAD). Of each tumor type, in agreement with the ethical committee of the UMCU, formalin fixed, 532 paraffin embedded (FFPE) material of 9-10 tumor specimens and five healthy specimens was collected 
533 from the tissue biobank (research protocol 17-786). The cohort of HNSC tumors comprised tongue tumors

534 with a diameter of $10 \mathrm{~mm}$ or larger. Only melanomas with a Breslow thickness of $0.5 \mathrm{~mm}$ or higher were

535 included. Tissue was obtained from primary tumors. Patients did not receive any systemic treatment or

536 radiotherapy before the tumor specimens were obtained. Healthy specimens were preferably separate

537 tissue blocks obtained from the same patients the tumor specimens were obtained from. In case of 538 glioblastoma, healthy specimens were collected post-mortem.

\section{Immunohistochemistry}

541 The following stainings were performed on consecutive slides of all tumor specimens: H\&E, Masson's

542 Trichrome, LAIR-1, NC410, CD45, CD3, CD68, and CD163. The LAIR-1, CD45, CD3, CD68, and CD163

543 stainings were performed using a Ventana Bench Mark XT Autostainer (Ventana Medical Systems, Tucson,

544 AZ, USA). Stainings were performed on $4 \mu \mathrm{m}$ sections of each tissue block. NC410 was biotinylated and 545 used for manual, immunohistochemical staining. Antigen retrieval was performed by incubating the slides 546 at $100^{\circ} \mathrm{C}$ for 24 or 64 mins in either EDTA or Citrate buffer, as indicated in the table below. For all assays,

547 the sections were incubated with the primary antibody for 1 hour at RT. Subsequently, the sections were

548 incubated with HRP-labeled secondary antibody, developed using $\mathrm{H} 2 \mathrm{O} 2$ and DAB and counterstained with 549 Hematoxylin. 


\begin{tabular}{|l|l|l|l|l|l|}
\hline Antibody & Supplier & Clone & Lot no & Pretreatment & Dilution \\
\hline $\begin{array}{l}\text { LAIR-1 } \\
\text { (polyclonal) }\end{array}$ & $\begin{array}{l}\text { ATLAS } \\
\text { antibodies }\end{array}$ & - & A103834 & 24' Citrate pH6.0 & $1: 500$ \\
\hline $\begin{array}{l}\text { LAIR-1 } \\
\text { (monoclonal) }\end{array}$ & $\begin{array}{l}\text { BD } \\
\text { Pharmingen }\end{array}$ & DX26 & 550810 & $64^{\prime}$ EDTA pH9.0 & $1: 100$ \\
\hline NC410 & Nextcure & - & - & $24^{\prime}$ Citrate pH6.0 & $1: 500$ \\
\hline CD45 & DAKO & PD7/26+2B11 & 110 & 24' EDTA pH9.0 & $1: 25$ \\
\hline CD3 & DAKO & - & 20042625 & 24' EDTA pH9.0 & $1: 100$ \\
\hline CD68 & Novocastra & KP1 & 6009203 & 24' EDTA pH9.0 & $1: 1600$ \\
\hline CD163 & Novocastra & $10 D 6$ & 6064616 & 24' EDTA pH9.0 & $1: 800$ \\
\hline
\end{tabular}

\section{Immunohistochemistry analysis}

557 All slides were digitalized using the Aperio Scanscope XT slide scanner. Evaluation of stained tissue slides

558 was performed using QuPath (version 0.2.0) software. The percentage of tumor tissue binding NC410 was

559 calculated by annotation of the tumor in the tissue slide and quantifying the percentage of DAB-stained

560 tissue by using the Pixel Classifier.

561 Scoring of the immune phenotype was based on the presence and distribution of CD3 positive

562 lymphocytes(61). Tumors with $\mathrm{CD}^{+}$cells in the tumor fields were scored "inflamed"; tumors with $\mathrm{CD}^{+}$

563 cells in their stroma, but without or with a relatively low amount of $\mathrm{CD}^{+}$in the tumor fields were scored

564 "immune excluded"; tumors with a lack of $\mathrm{CD}^{+}$cells in their stroma as well as in their tumor fields were

565 scored "immune desert". 
566 For quantification of the immune cell counts, regions of interest (ROIs) were annotated on the tissue slides

567 by drawing circles with a diameter of $600 \mu \mathrm{m}$ at five random spots within the NC410 binding part of the

568 tumor. Positive cells were quantified using the Positive Cell Detection tool. For each staining, stain vectors

569 and DAB cutoffs were determined based on a representative slide; the settings were kept the same for all

570 slides. Tumor area within the circles that was lost during staining procedure or that was negative for

571 NC410 binding was excluded from the analysis.

572 The percentage of NC410 binding to tissue within a tumor was calculated by dividing the stained area by

573 the total tumor area. The immune cell counts for each tumor were calculated by dividing the total number

574 of positive cells within the five ROls by the total surface in $\mathrm{mm}^{2}$ of these ROls.

576 Statistics

577 Data were graphed and analyzed using GraphPad Prism 8.0 (Graph Pad software). Data are presented as

578 the mean \pm standard error of the mean (SEM). The significance was analyzed with unpaired, two-tailed

579 Student's t-tests and 2-way ANOVA followed by multiple comparison.

581 Acknowledgments

582 We thank Michiel van der Vlist for his critical comments on the manuscript.

\section{Competing interests}

$585 \mathrm{LT}, \mathrm{CS}, \mathrm{AP}, \mathrm{JS}, \mathrm{JB}, \mathrm{ZC}, \mathrm{LL}, \mathrm{SL}$ and DF are employees from Nextcure. Nextcure holds a patent on NC410. 
Conceptualization: ZC, LL, SL, SW, DF, LM

\section{Data curation: JS}

Formal analysis: MIPR, LT, EJR, CS, AP, AS, EE, SVV, JS, LL, SL, SW, DF, LM

Funding acquisition: DF, LM

Investigation: MIPR, LT, EJR, CS, AP, AS, EE, SVV, JS, JB

Methodology: MIPR, LT

Project administration: ZC

Supervision: DF, LM

Writing - original draft: MIPR, LT

Writing - review \& editing: MIPR, LT, EJR, CS, AP, AS, EE, SVV, JS, JB, ZC, LL, SL, SW, DF, LM

\section{Figure Legends}

Figure1. Collagen and LAIR-1 are overexpressed in tumors and correlate with poor overall survival while

A) The expression of 43 collagen genes, LAIR-1 and LAIR-2 in normal (grey) and tumor tissue (red) were 
considered individuals with low expression and those in the upper quantile (red) were considered those with high expression respectively. The estimate of overall survival was determined using Kaplan-Meier LAIR-2 mRNA expression for overall survival analysis.

\section{Figure 2. NC410 is an Fc dimeric fusion protein that blocks LAIR-1 interaction with collagen.}

611 A) NC410 is a biologic resulting by fusing LAIR-2 with a functional IgG1 to generate a dimeric fusion protein. analysis. C) Indicated amounts of collagen I were plate coated and the binding of soluble LAIR-1 was inhibited by NC410. Asterisks indicate statistical significance (****P $<0.0001,2$-way ANOVA). D and E) reporter. LAIR-1 ligation and CD3 ligation induce NFAT-GFP signaling. A parental cell line containing the

620 cells is shown over time. Points represent the median of $n=3$ (with experimental triplicates in each

621 independently performed experiment) and the whiskers indicate the $95 \%$ confidence interval (Cl). Isotype

622 control was used at the highest concentration $(100 \mathrm{ug} / \mathrm{mL})$ and showed no inhibition of NFAT-GFP

623 signaling. Anti-human LAIR (8A8 clone) and anti-mouse CD3 were used as positive controls. Closed circles

624 in E) indicate NC410 treatment and open circles indicates control treatment. Significant differences

625 between different treatment groups of hLAIR-1 reporter cells are indicated (and tested using a two-way

626 ANOVA with Dunnett's correction). In all plots: * $\mathrm{P} \leq 0.05, * * \mathrm{P} \leq 0.01, * * * \mathrm{P} \leq 0.001, * * * * \mathrm{P} \leq 0.0001$. 
Figure 3. NC410 therapy promotes T cell expansion and tumor regression.

A) Model for analysis of T cell expansion and xeno-anti-tumor response in the presence of NC410 or control Fc. $1.5 \times 10^{7}$ total human PBMCs were adoptively transferred by intravenous injection to NSG mouse on Day 0. $2 \times 10^{5}$ P815 tumor cells were injected subcutaneously on Day 1 . Mice were treated on Days 1 and 3 with NC410 or control lgG1 (1 mg/kg) by intraperitoneal injection ( $\mathrm{N}=6 /$ group). B) CD $8^{+} \mathrm{T}$ cell expansion in blood on day 6 and 13. Asterisks indicate statistical significance ( ${ }^{* *} P<0.01,2$-way ANOVA with Sidak multiple comparisons). C) P815 tumor was measured every 2 - 3 days with a caliper and tumor volume was calculated. Asterisks indicate statistical significance $\left({ }^{*} P<0.05,{ }^{*} P<0.01,2\right.$-way ANOVA with Sidak multiple comparisons). D) Humanized tumor model of HT-29 tumor injected subcutaneously in the 637 presence of human PBMCs. $2 \times 10^{7}$ total human PBMCs were adoptively transferred intraperitoneally (E) or intravenously (F) to NSG mice ( $\mathrm{N}=6 /$ group) on Day $0.1 \times 10^{6} \mathrm{HT}-29$ tumor cells were injected subcutaneously with Matrigel on Day 1 . Mice were treated with NC410 or control by intraperitoneal

640 injection, Q4D x4 doses followed by Q7D until endpoint. Tumor growth was monitored 1 - 3 times a week

642 significance ( $* * * * \mathrm{P}<0.0001,2$-way ANOVA with Sidak multiple comparisons). F) Dose dependent effect 643 of NC410. 6 mice per group were used for all experiments. Asterisks indicate statistical significance $\left({ }^{*} \mathrm{P}<\right.$ $0.05, * * * \mathrm{P}<0.001,2$-way ANOVA with Tukey multiple comparisons). G) In vitro chromium release assay after 24h using HT-29 and PBMCs at 3 different effector to target ratios. 20:1 and 50:1 n=16 and 100:1

$646 \mathrm{n}=25$ in 17 independently performed experiments. Closed circles indicate NC410 treatment and open 647 circles indicates control treatment. 

$\mathrm{CD}^{+}$TIL cell numbers in the tumor. The cell number was counted by flow cytometry and normalized to weight (gram) of tumor tissue. Asterisks indicate statistical significance ( ${ }^{*} \mathrm{P}<0.05$, two-tailed t-test). C)

657 Cytokine production by TILs following ex vivo restimulation with PMA and ionomycin for 5 hours. Cells 658 were intracellularly stained for TNF- $\alpha$ and IFN- $\gamma$ and the indicated cell populations were counted by flow 659 cytometry. The data presents the cell counts normalized to the weight (gram) of the tumor tissue. Asterisks indicate statistical significance ( ${ }^{*} \mathrm{P}<0.05$, two-tailed t-test). D-E) Analysis of tumor and spleen

661 for cytokines (D) and chemokines (E) for analysis of local and systemic effects respectively. Tissue lysate 662 protein was extracted from the tumor and spleen tissues. The cytokines were analyzed by Luminex and 663 presented as the relative levels normalized to weight (gram) of tissue. Asterisks indicate statistical significance $\left({ }^{*} \mathrm{P}<0.05,{ }^{*} \mathrm{P}<0.01\right.$, two-tailed t-test).

Figure 5. NC410 increases MMP2 and Granzyme B derived collagen fragments 
673 serum at baseline, at week 1, 2,3 and 4. MMP-2 derived collagen VI and granzyme B derived collagen IVI

674 degradation products were increased at week 4 after NC410 administration. Closed circles indicate NC410

675 treatment and open circles indicates control treatment. Asterisks indicate statistical significance $(* * * * \mathrm{P}$

$676<0.0001,2$-way ANOVA followed with Sidak multiple comparisons).

678 Figure 6- NC410 binds to collagen rich tumors with an immune excluded phenotype

679 A) Schematic representation of the immunohistochemical stainings performed. B) Representative 680 hematoxylin and eosin (H\&E), Masson Trichrome, NC410 and LAIR-1 staining in a stomach tumor 681 specimen. C) Quantification of LAIR-1 and NC410 staining of 10 patients per tumor type across 7 different 682 tumor types (Head and neck (HNSC), melanoma, non-small cell lung carcinoma (NSCLC), ovarian, 683 pancreatic (PDAC) and stomach cancer (STAD)). In the associated piecharts, we show the percentage of 684 NC410 binding where green indicates the percentage of NC410 positive area and red indicates the 685 percentage of NC410 negative area. D) Higher magnification pictures of stomach cancer specimens show 686 LAIR-1 ${ }^{+}$cells (depicted in red, right side) colocalizing with NC410 positive areas (depicted in red, left side). 687 E) Patients within each tumor type were characterized into immune desert, immune excluded and 688 immune inflamed based on $\mathrm{CD}^{+} \mathrm{T}$ cell presence and localization. F) Quantification of NC410 staining 689 across the different immune phenotypes in the 7 different tumor types analyzed.

Supplemental Figure 1- NC410 blocks LAIR-1 functional interaction with collagen.

692 NC410 dose response measured by incucyte imaging during $24 \mathrm{~h}$. Representative microscopic images $693(10 x)$ of WT and hLAIR-1 reporter cells over time. NC410 protein was added at increasing concentrations 694 and inhibited collagen I (5 ug/mL) mediated NFAT-GFP signaling through LAIR-1 binding. Isotype control 
was used at the highest concentration $(100 \mathrm{ug} / \mathrm{mL})$ and showed no inhibition of NFAT-GFP signaling.

Pictures were taken every $1 \mathrm{~h}$ for $24 \mathrm{~h}$. Representative images from 1 out of 3 independently performed experiments (each with experimental triplicates).

\section{Supplemental Figure 2- HT-29 mRNA collagen expression by RNA sequencing}

For collagen gene expression analysis of HT-29 cells, data was acquired from the public dataset GSE41586 and normalized using the DESeq2 package (v1.28.1) in R (v4.0.2). Data was then log2 transformed and plotted using the ggplot package (v3.3.2).

Immunofluorescence analysis of HT-29 cells stained with A) isotype control, B) NC410 or C) pan-collagen

707 antibody. 40x magnification D) HT-29 cells were removed from culture flasks using increasing concentrations of EDTA for 10 min. MFI of NC410 staining is shown, demonstrating that EDTA treated HT-

710 binding. Data from 3 independently performed experiments.

\section{Supplemental Figure 4- Chemokine expression in a humanized HT-29 tumor model}

CXCL10, CXCL11 and CXCL12 chemokine expression correlate with reduced tumor growth at day 27 after

714 HT-29 injection. P value was calculated by linear regression with F test. 6 mice per group were used. Black 715 circles indicate NC410 treatment and open circles indicates control treatment. 
718 different tumor types (Head and neck (HNSC), melanoma, -non-small cell lung carcinoma (NSCLC), ovarian, pancreatic (PDAC) and stomach cancer (STAD))

\section{Supplemental Figure 6- Immunohistochemical analysis of healthy tissue} tissue matching the tumors used in Supplemental Figure 5.

\section{Supplemental Figure 7- Characterization of LAIR-1+ cells in primary human tumors}

A) Representative LAIR-1 FC, NC410, CD45, CD3, CD68 and CD163 staining in a stomach tumor specimen.

732 (STAD)). 
bioRxiv preprint doi: https://doi.org/10.1101/2020.10.21.349480; this version posted October 22, 2020. The copyright holder for this preprint (which was not certified by peer review) is the author/funder, who has granted bioRxiv a license to display the preprint in perpetuity. It is made available under aCC-BY 4.0 International license.

\section{Supplemental Figure 8- Tumor immune phenotyping}

737 Representative image of the 3 different immune phenotypes, immune desert, immune excluded and

738 immune inflamed in an ovarian tumor specimen.

739

740

741

742

743

744

745

746

747

748

749

750

751

752

753 


\section{References}

755

756

757

758

759

760

761

762

763

764

765

766

767

768

769

770

771

772

773

774

775

776

777

778

779

780

781

782

783

784

785

786

787

788

789

790

791

792

793

794

795

796

797

798

799

800

1. Darvin P, Toor SM, Nair VS, Elkord E. Immune checkpoint inhibitors: recent progress and potential biomarkers. Experimental and Molecular Medicine. 2018;50.

2. Gibney GT, Weiner LM, Atkins MB. Predictive biomarkers for checkpoint inhibitor-based immunotherapy. Lancet Oncology. 2016;17(12):E542-E51.

3. Bonnans $\mathrm{C}$, Chou J, Werb Z. Remodelling the extracellular matrix in development and disease. Nat Rev Mol Cell Bio. 2014;15(12):786-801.

4. Myllyharju J, Kivirikko KI. Collagens, modifying enzymes and their mutations in humans, flies and worms. Trends in Genetics. 2004;20(1):33-43.

5. Hynes RO. The extracellular matrix: not just pretty fibrils. Science. 2009;326(5957):1216-9.

6. Fang M, Yuan JP, Peng CW, Li Y. Collagen as a double-edged sword in tumor progression. Tumor Biol. 2014;35(4):2871-82.

7. Naba A, Clauser KR, Hoersch S, Liu H, Carr SA, Hynes RO. The Matrisome: In Silico Definition and In Vivo Characterization by Proteomics of Normal and Tumor Extracellular Matrices. Molecular \& Cellular Proteomics. 2012;11(4).

8. Lu PF, Weaver VM, Werb Z. The extracellular matrix: A dynamic niche in cancer progression. Journal of Cell Biology. 2012;196(4):395-406.

9. Levental KR, Yu HM, Kass L, Lakins JN, Egeblad M, Erler JT, et al. Matrix Crosslinking Forces Tumor Progression by Enhancing Integrin Signaling. Cell. 2009;139(5):891-906.

10. Naba A, Clauser KR, Whittaker CA, Carr SA, Tanabe KK, Hynes RO. Extracellular matrix signatures of human primary metastatic colon cancers and their metastases to liver. Bmc Cancer. 2014;14.

11. Kalluri R, Zeisberg M. Fibroblasts in cancer. Nature Reviews Cancer. 2006;6(5):392-401.

12. Kalluri R. The biology and function of fibroblasts in cancer. Nature Reviews Cancer. 2016;16(9):582-98.

13. Naba A, Clauser KR, Lamar JM, Carr SA, Hynes RO. Extracellular matrix signatures of human mammary carcinoma identify novel metastasis promoters. Elife. 2014;3.

14. Januchowski R, Zawierucha P, Rucinski M, Nowicki M, Zabel M. Extracellular Matrix Proteins Expression Profiling in Chemoresistant Variants of the A2780 Ovarian Cancer Cell Line. Biomed Research International. 2014.

15. Antonio N, Bonnelykke-Behrndtz ML, Ward LC, Collin J, Christensen IJ, Steiniche T, et al. The wound inflammatory response exacerbates growth of pre-neoplastic cells and progression to cancer. Embo Journal. 2015;34(17):2219-36.

16. Rosowski EE, Huttenlocher A. Neutrophils, Wounds, and Cancer Progression. Developmental Cell. 2015;34(2):134-6.

17. Henke E, Nandigama R, Ergun S. Extracellular Matrix in the Tumor Microenvironment and Its Impact on Cancer Therapy. Front Mol Biosci. 2020;6.

18. Rygiel TP, Stolte EH, de Ruiter T, van de Weijer ML, Meyaard L. Tumor-expressed collagens can modulate immune cell function through the inhibitory collagen receptor LAIR-1. Mol Immunol. 2011;49(1-2):402-6.

19. Bulla R, Tripodo C, Rami D, Ling GS, Agostinis C, Guarnotta C, et al. C1q acts in the tumour microenvironment as a cancer-promoting factor independently of complement activation. Nature Communications. 2016;7.

20. Meyaard L, Adema GJ, Chang C, Woollatt E, Sutherland GR, Lanier LL, et al. LAIR-1, a novel inhibitory receptor expressed on human mononuclear leukocytes. Immunity. 1997;7(2):283-90.

21. Lebbink RJ, de Ruiter T, Adelmeijer J, Brenkman AB, van Helvoort JM, Koch M, et al. Collagens are functional, high affinity ligands for the inhibitory immune receptor LAIR-1. J Exp Med.

2006;203(6):1419-25. 
22. Son M, Santiago-Schwarz F, Al-Abed Y, Diamond B. C1q limits dendritic cell differentiation and activation by engaging LAIR-1. Proceedings of the National Academy of Sciences of the United States of America. 2012;109(46):E3160-7.

23. Nordkamp MJMO, van Eijk M, Urbanus RT, Bont L, Haagsman HP, Meyaard L. Leukocyteassociated Ig-like receptor-1 is a novel inhibitory receptor for surfactant protein D. Journal of Leukocyte Biology. 2014;96(1):105-11.

24. Meyaard L, Adema GJ, Chang C, Woollatt E, Sutherland GR, Lanier LL, et al. LAIR-1, a novel inhibitory receptor expressed on human mononuclear leukocytes. Immunity. 1997;7:283-90.

25. Maasho K, Masilamani M, Valas R, Basu S, Coligan JE, Borrego F. The inhibitory leukocyteassociated Ig-like receptor-1 (LAIR-1) is expressed at high levels by human naive T cells and inhibits TCR mediated activation. Mol Immunol. 2005;42(12):1521-30.

26. Zhang Y, Lv K, Zhang CM, Jin BQ, Zhuang R, Ding Y. The role of LAIR-1 (CD305) in T cells and monocytes/macrophages in patients with rheumatoid arthritis. Cell Immunol. 2014;287(1):46-52.

27. Park JE, Brand DD, Rosloniec EF, Yi AK, Stuart JM, Kang AH, et al. Leukocyte-associated immunoglobulin-like receptor 1 inhibits T-cell signaling by decreasing protein phosphorylation in the Tcell signaling pathway. Journal of Biological Chemistry. 2020;295(8):2239-47.

28. van der Vuurst de Vries AR, Clevers $H$, Logtenberg T, Meyaard L. Leukocyte-associated immunoglobulin-like receptor-1 (LAIR-1) is differentially expressed during human B cell differentiation and inhibits B cell receptor-mediated signaling. Eur J Immunol. 1999;29(10):3160-7.

29. Merlo A, Tenca C, Fais F, Battini L, Ciccone E, Grossi CE, et al. Inhibitory receptors CD85j, LAIR-1, and CD152 down-regulate immunoglobulin and cytokine production by human B lymphocytes. Clin Diagn Lab Immunol. 2005;12(6):705-12.

30. Son M, Diamond B. C1q-mediated repression of human monocytes is regulated by leukocyteassociated Ig-like receptor 1 (LAIR-1). Mol Med. 2015;20:559-68.

31. Son M, Santiago-Schwarz F, Al-Abed Y, Diamond B. C1q limits dendritic cell differentiation and activation by engaging LAIR-1. Proc Natl Acad Sci U S A. 2012.

32. Poggi A, Tomasello E, Ferrero E, Zocchi MR, Moretta L. p40/LAIR-1 regulates the differentiation of peripheral blood precursors to dendritic cells induced by granulocyte-monocyte colony-stimulating factor. Eur J Immunol. 1998;28(7):2086-91.

33. Olde Nordkamp MJ, van Roon JA, Douwes M, de Ruiter T, Urbanus RT, Meyaard L. Enhanced secretion of leukocyte-associated immunoglobulin-like receptor 2 (LAIR-2) and soluble LAIR-1 in rheumatoid arthritis: LAIR-2 is a more efficient antagonist of the LAIR-1-collagen inhibitory interaction than is soluble LAIR-1. Arthritis Rheum. 2011;63(12):3749-57.

34. Meyaard L, van den Berg M, de Ruiter T, Raynal N, van Roon J, Lenting P, et al. Leukocyteassociated immunoglobulin-like receptor (LAIR)-2 is a soluble competitor of the collagen/LAIR-1 inhibitory immune interaction. Faseb Journal. 2008;22.

35. Nissen NI, Karsdal M, Willumsen N. Collagens and Cancer associated fibroblasts in the reactive stroma and its relation to Cancer biology. J Exp Clin Canc Res. 2019;38.

36. Xu SS, Xu HX, Wang WQ, Li S, Li H, Li TJ, et al. The role of collagen in cancer: from bench to bedside. J Transl Med. 2019;17(1).

37. Ke ZF, He WL, Lai YH, Guo XF, Chen SR, Li SH, et al. Overexpression of Collagen Triple Helix Repeat Containing 1 (CTHRC1) is associated with tumour aggressiveness and poor prognosis in human non-small cell lung cancer. Oncotarget. 2014;5(19):9410-24.

38. lizasa $\mathrm{T}$, Chang $\mathrm{H}$, Suzuki $\mathrm{M}$, Otsuji M, Yokoi S, Chiyo $\mathrm{M}$, et al. Overexpression of collagen XVIII is associated with poor outcome and elevated levels of circulating serum endostatin in non-small cell lung cancer. Clinical Cancer Research. 2004;10(16):5361-6.

39. Huang HP, Li TT, Ye GT, Zhao LY, Zhang ZZ, Mo DB, et al. High expression of COL10A1 is associated with poor prognosis in colorectal cancer. Oncotargets Ther. 2018;11:1571-81. 
40. Wu YH, Chang TH, Huang YF, Huang HD, Chou CY. COL11A1 promotes tumor progression and predicts poor clinical outcome in ovarian cancer. Oncogene. 2014;33(26):3432-40.

41. Cheon DJ, Tong YG, Sim MS, Dering J, Berel D, Cui XJ, et al. A Collagen-Remodeling Gene Signature Regulated by TGF-beta Signaling Is Associated with Metastasis and Poor Survival in Serous Ovarian Cancer. Clinical Cancer Research. 2014;20(3):711-23.

42. Lebbink RJ, van den Berg MCW, de Ruiter T, Raynal N, van Roon JAG, Lenting PJ, et al. The soluble leukocyte-associated Ig-like receptor (LAIR)-2 antagonizes the Collagen/LAIR-1 inhibitory immune interaction. Journal of Immunology. 2008;180(3):1662-9.

43. Waldmann TA. Cytokines in Cancer Immunotherapy. Csh Perspect Biol. 2018;10(12).

44. Strieter RM. Chemokines: Not just leukocyte chemoattractants in the promotion of cancer. Nature Immunology. 2001;2(4):285-6.

45. Sund M, Kalluri R. Tumor stroma derived biomarkers in cancer. Cancer Metast Rev. 2009;28(12):177-83.

46. Rolff HC, Christensen IJ, Vainer B, Svendsen LB, Eefsen RL, Wilhelmsen M, et al. The Prognostic and Predictive Value of Soluble Type IV Collagen in Colorectal Cancer: A Retrospective Multicenter Study. Clinical Cancer Research. 2016;22(10):2427-34.

47. Jensen C, Madsen DH, Hansen M, Schmidt H, Svane IM, Karsdal MA, et al. Non-invasive biomarkers derived from the extracellular matrix associate with response to immune checkpoint blockade (anti-CTLA-4) in metastatic melanoma patients. Journal for Immunotherapy of Cancer. 2018;6. 48. Binnewies M, Roberts EW, Kersten K, Chan V, Fearon DF, Merad M, et al. Understanding the tumor immune microenvironment (TIME) for effective therapy. Nature Medicine. 2018;24(5):541-50. 49. Chen DS, Mellman I. Oncology Meets Immunology: The Cancer-Immunity Cycle. Immunity. 2013;39(1):1-10.

50. Beatty GL, Gladney WL. Immune Escape Mechanisms as a Guide for Cancer Immunotherapy. Clinical Cancer Research. 2015;21(4):687-92.

51. Cox TR, Erler JT. Molecular Pathways: Connecting Fibrosis and Solid Tumor Metastasis. Clinical Cancer Research. 2014;20(14):3637-43.

52. Pickup MW, Mouw JK, Weaver VM. The extracellular matrix modulates the hallmarks of cancer. Embo Reports. 2014;15(12):1243-53.

53. Salmon H, Franciszkiewicz K, Damotte D, Dieu-Nosjean MC, Validire P, Trautmann A, et al. Matrix architecture defines the preferential localization and migration of T cells into the stroma of human lung tumors. Journal of Clinical Investigation. 2012;122(3):899-910.

54. Hartmann N, Giese NA, Giese T, Poschke I, Offringa R, Werner J, et al. Prevailing Role of Contact Guidance in Intrastromal T-cell Trapping in Human Pancreatic Cancer. Clinical Cancer Research. 2014;20(13):3422-33.

55. Kuczek DE, Larsen AMH, Thorseth ML, Carretta M, Kalvisa A, Siersbaek MS, et al. Collagen density regulates the activity of tumor-infiltrating T cells. Journal for Immunotherapy of Cancer. 2019;7. 56. Kehlet SN, Sanz-Pamplona R, Brix S, Leeming DJ, Karsdal MA, Moreno V. Excessive collagen turnover products are released during colorectal cancer progression and elevated in serum from metastatic colorectal cancer patients. Sci Rep-Uk. 2016;6.

57. Bager CL, Willumsen N, Leeming DJ, Smith V, Karsdal MA, Dornan D, et al. Collagen degradation products measured in serum can separate ovarian and breast cancer patients from healthy controls: $\mathrm{A}$ preliminary study. Cancer Biomarkers. 2015;15(6):783-8.

58. Willumsen N, Bager CL, Leeming DJ, Smith V, Karsdal MA, Dornan D, et al. Extracellular matrix specific protein fingerprints measured in serum can separate pancreatic cancer patients from healthy controls. Bmc Cancer. 2013;13.

59. Havel JJ, Chowell D, Chan TA. The evolving landscape of biomarkers for checkpoint inhibitor immunotherapy. Nature Reviews Cancer. 2019;19(3):133-50. 
897 60. Trujillo JA, Sweis RF, Bao R, Luke JJ. T Cell-Inflamed versus Non-T Cell-Inflamed Tumors: A 898 Conceptual Framework for Cancer Immunotherapy Drug Development and Combination Therapy 899 Selection. Cancer Immunology Research. 2018;6(9):990-1000.

900 61. Chen DS, Mellman I. Elements of cancer immunity and the cancer-immune set point. Nature.

901 2017;541(7637):321-30.

902 62. Jiang P, Gu SQ, Pan D, Fu JX, Sahu A, Hu XH, et al. Signatures of T cell dysfunction and exclusion 903 predict cancer immunotherapy response. Nature Medicine. 2018;24(10):1550-+.

904 63. Xu LJ, Wang SL, Li JF, Li J, Li BY. Cancer immunotherapy based on blocking immune suppression 905 mediated by an immune modulator LAIR-1. Oncoimmunology. 2020;9(1).

906 64. Charan J, Kantharia ND. How to calculate sample size in animal studies? J Pharmacol

907 Pharmacother. 2013;4(4):303-6.

908 65. Arifin WN, Zahiruddin WM. Sample Size Calculation in Animal Studies Using Resource Equation 909 Approach. Malays J Med Sci. 2017;24(5):101-5.

910 66. Brandsma AM, ten Broeke T, Nederend M, Meulenbroek LAPM, van Tetering G, Meyer S, et al. 911 Simultaneous Targeting of Fc gamma Rs and Fc alpha RI Enhances Tumor Cell Killing. Cancer Immunology 912 Research. 2015;3(12):1316-24.

913 67. Ronnow SR, Dabbagh RQ, Genovese F, Nanthakumar CB, Barrett VJ, Good RB, et al. Prolonged 914 Scar-in-a-Jar: an in vitro screening tool for anti-fibrotic therapies using biomarkers of extracellular matrix 915 synthesis. Resp Res. 2020;21(1). 


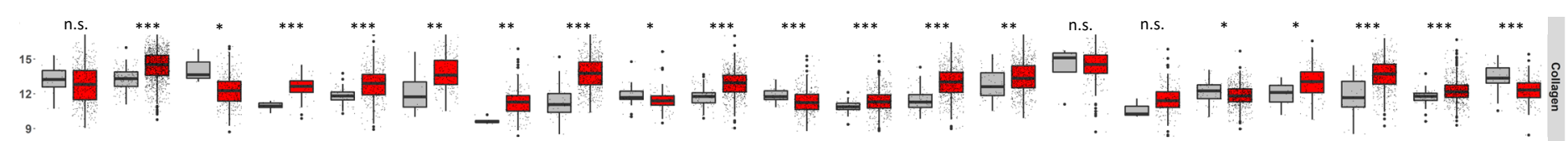
Fot abde

B

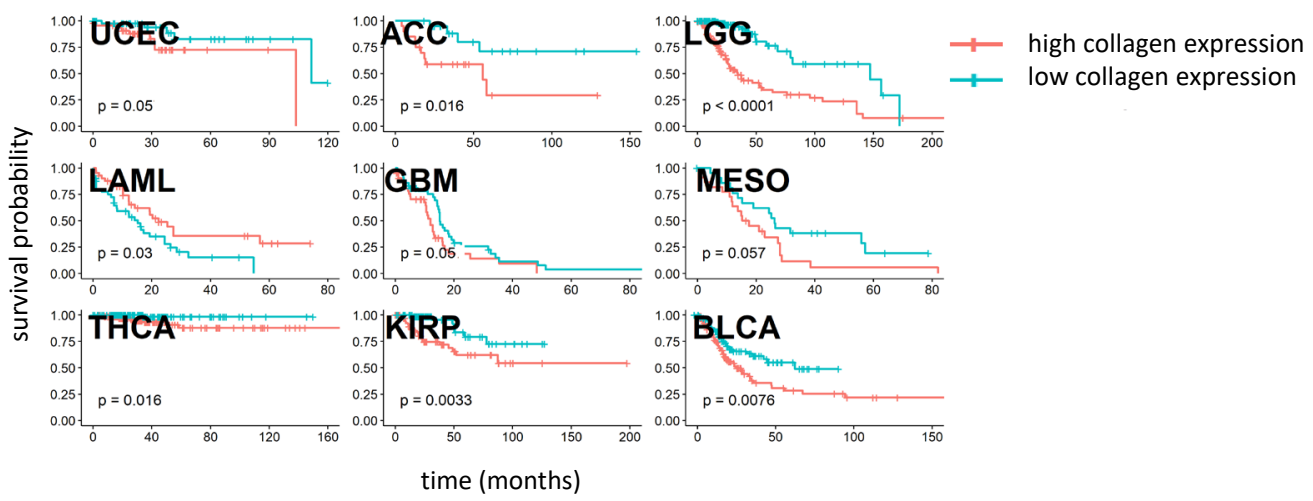

C

D
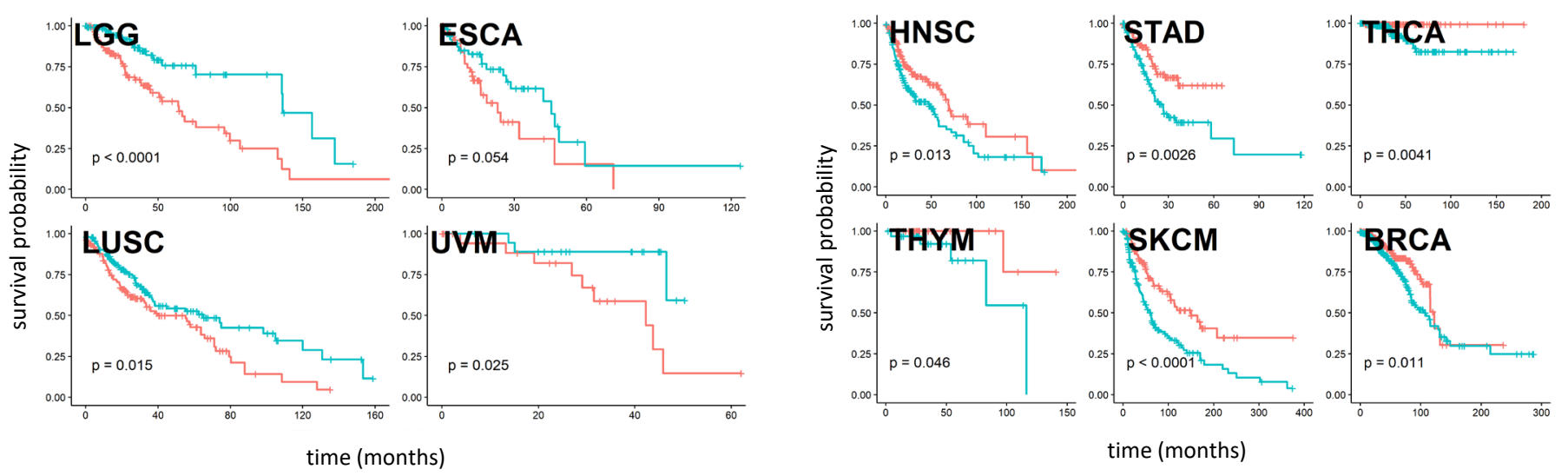
Figure 2- NC410 is an Fc dimeric fusion protein that blocks LAIR-1 interaction with collagen.

A)

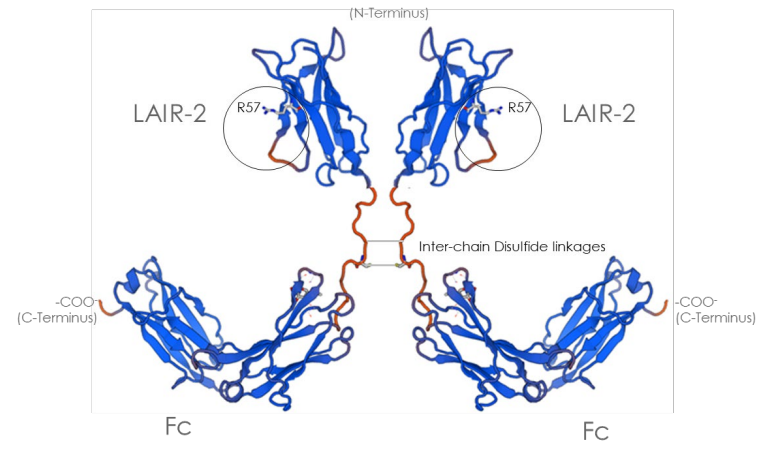

C)

Collagen I

$1 \mathrm{ug} / \mathrm{mL}$ LAIR-1 Fc Biotin $+10 \mathrm{ug} / \mathrm{mL}$ proteins

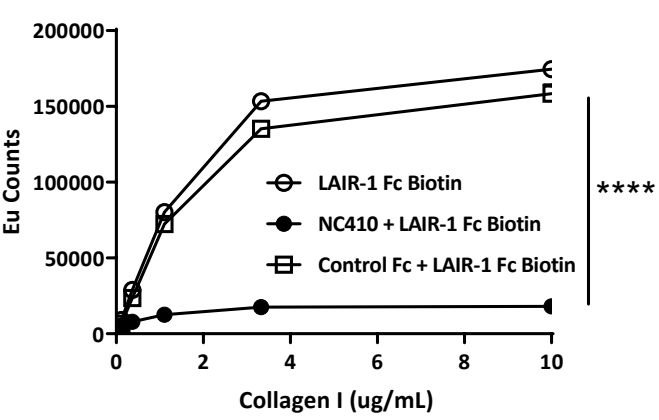

E)

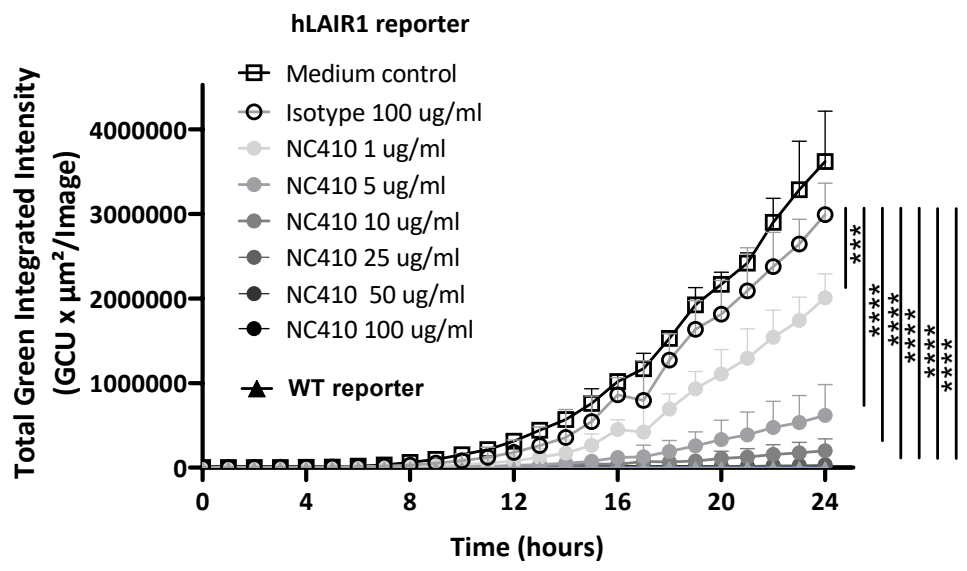

B)

\begin{tabular}{|c|c|c|c|c|c|c|}
\hline & $\frac{\text { Analysis }}{\text { Method: }}$ & Reagent & $\frac{\text { Apparent KD }}{\text { (M) }}$ & kon(1/Ms) & $\underline{\operatorname{ddis}(1 / s)}$ & Full $\mathbf{R}^{\wedge} \mathbf{2}$ \\
\hline \multirow[t]{2}{*}{ Human } & Molarity & hCollagen I & $1.26 \mathrm{E}-10$ & $1.50 \mathrm{E}+06$ & $1.86 \mathrm{E}-04$ & 0.9979 \\
\hline & Binding sites $/ \mathrm{mol}$ & hCollagen I & $1.72 \mathrm{E}-08$ & $1.08 \mathrm{E}+04$ & $1.84 \mathrm{E}-04$ & 0.9979 \\
\hline \multirow[t]{2}{*}{ Mouse } & Molarity & mCollagen I & $9.18 \mathrm{E}-11$ & $2.39 E+06$ & $1.94 \mathrm{E}-04$ & 0.9949 \\
\hline & Binding sites $/ \mathrm{mol}$ & mCollagen I & $1.25 \mathrm{E}-08$ & $1.76 \mathrm{E}+04$ & $1.94 \mathrm{E}-04$ & 0.9949 \\
\hline \multirow[t]{2}{*}{ Rat } & Molarity & rCollagen I & 3.87E-11 & $6.57 E+06$ & $2.41 \mathrm{E}-04$ & 0.9969 \\
\hline & Binding sites $/ \mathrm{mol}$ & rCollagen I & $3.74 \mathrm{E}-09$ & $6.61 E+04$ & $2.41 E-04$ & 0.9969 \\
\hline \multirow[t]{2}{*}{ Human } & Molarity & hCollagen III & 5.10E-10 & $4.88 \mathrm{E}+05$ & $2.41 E-04$ & 0.9959 \\
\hline & Binding sites $/ \mathrm{mol}$ & hCollagen III & 4.70E-08 & $5.30 E+03$ & $2.41 \mathrm{E}-04$ & 0.9959 \\
\hline \multirow[t]{2}{*}{ Mouse } & Molarity & mCollagen III & & & & \\
\hline & Binding sites $/ \mathrm{mol}$ & mCollagen III & & & & \\
\hline \multirow[t]{2}{*}{ Rat } & Molarity & rCollagen III & $8.78 \mathrm{E}-10$ & $8.65 E+05$ & 4.33E-04 & 0.9955 \\
\hline & Binding sites $/ \mathrm{mol}$ & rCollagen III & $1.15 \mathrm{E}-07$ & $6.60 E+03$ & $4.32 \mathrm{E}-04$ & 0.9955 \\
\hline
\end{tabular}

D)

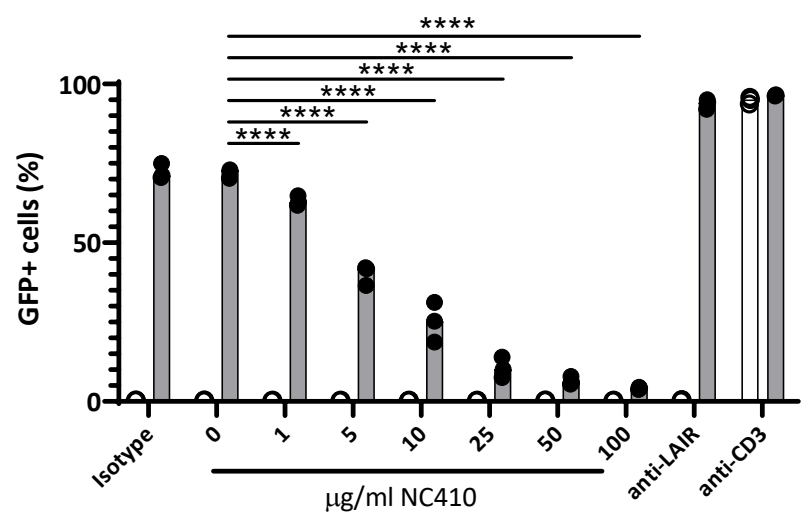

- WT reporter

- hLAIR1 reporter 
Figure 3-NC410 therapy promotes T cell expansion and tumor regression.

A)

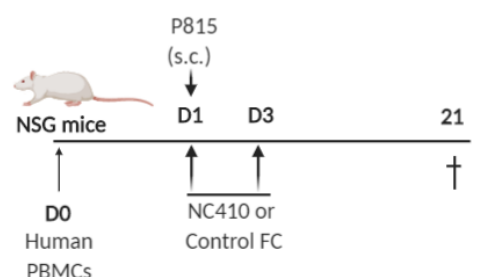

(i.v.)

B)

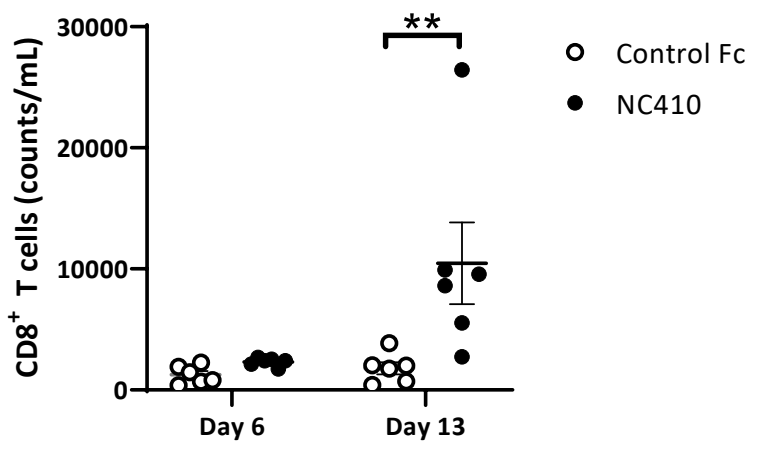

Days post inoculation

D)

$$
\text { F) }
$$

F)

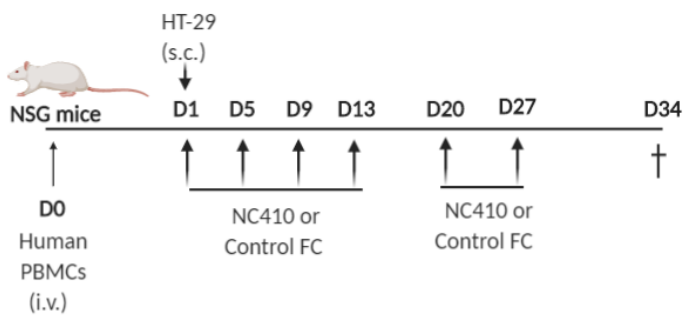

H)

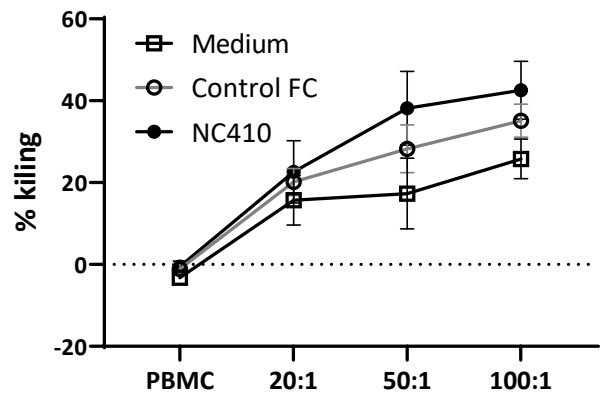

C)

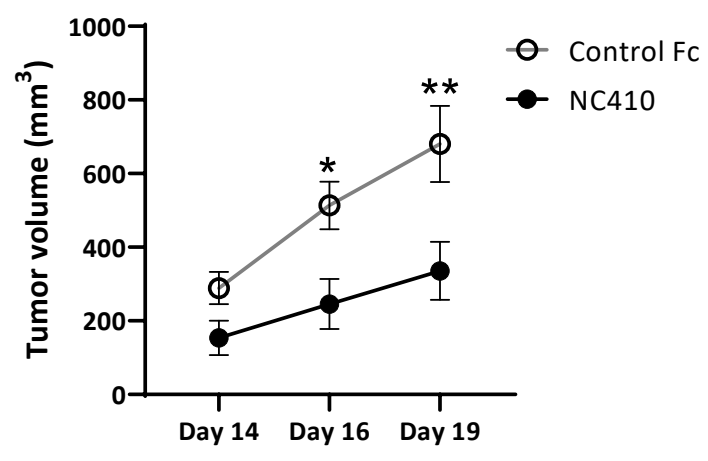

Days post inoculation

E)

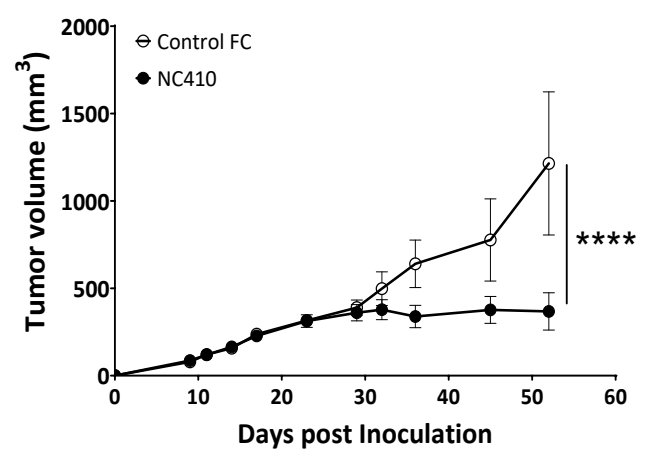

G)

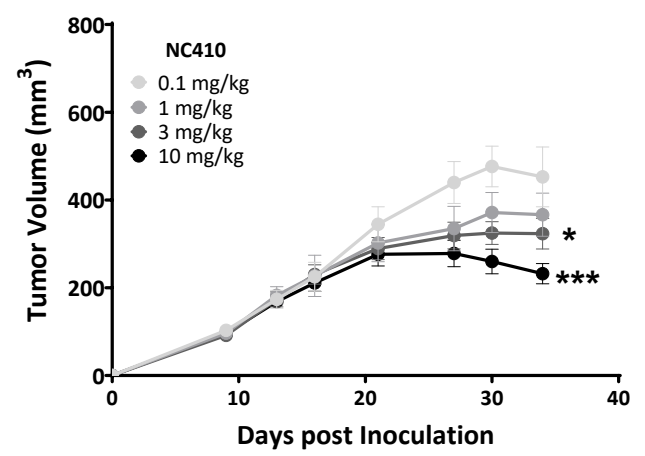


Figure 4- NC410 therapy induces immune activity in the local tumor microenvironment

A)

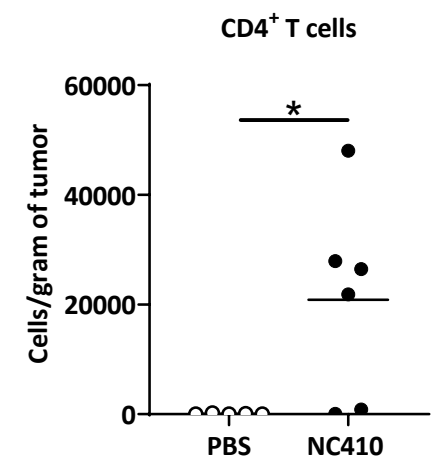

D)

Spleen

IFN- $\gamma$
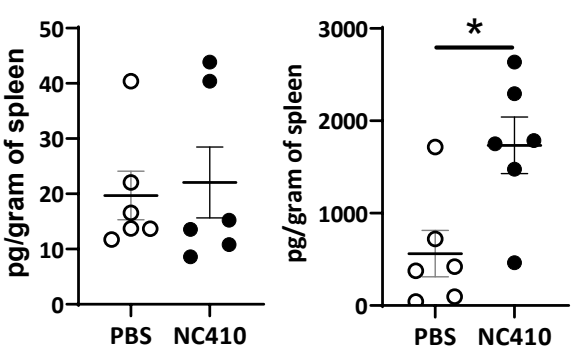

Tumor
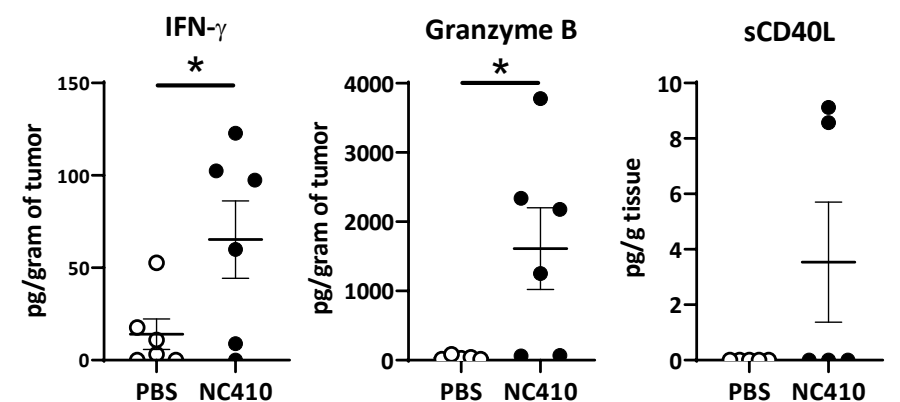

B)

CD8 $^{+} T$ Cells

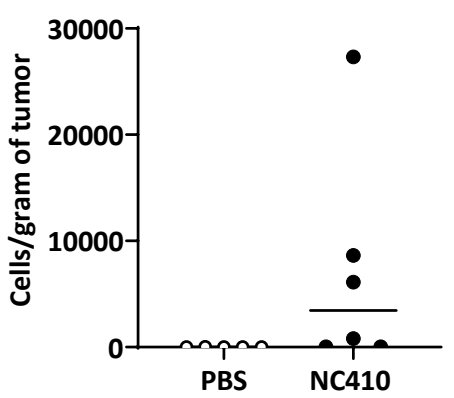

E)
C)

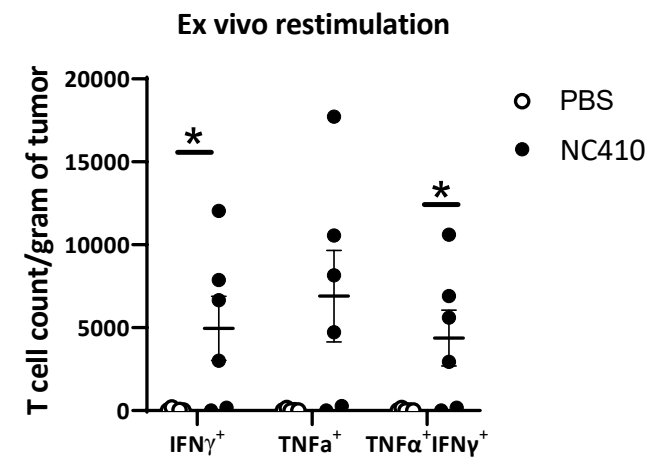

\section{Spleen}

CXCL10
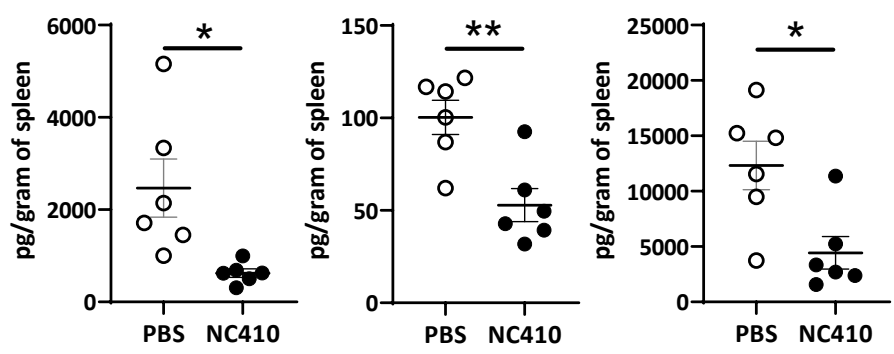

Tumor
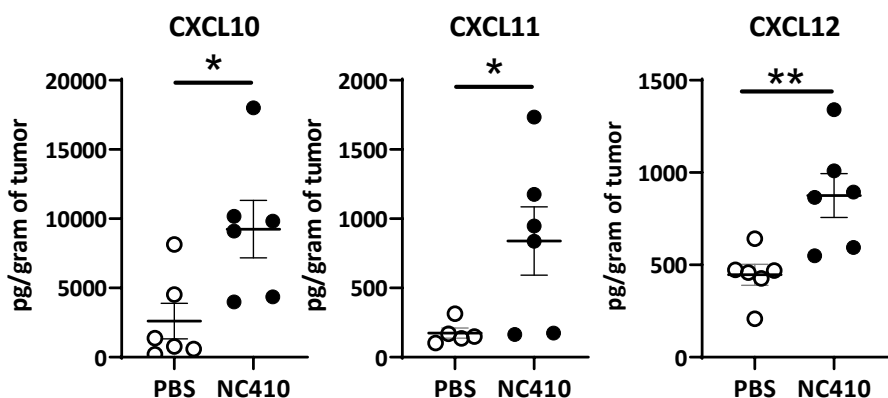
A)

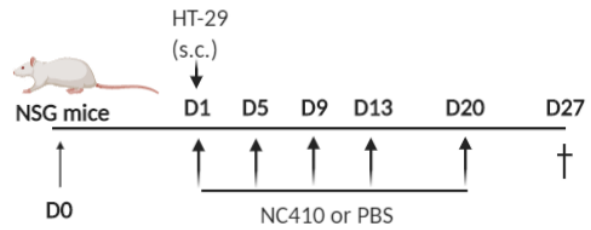

Human

PBMCs

(i.v.)

B)

C)

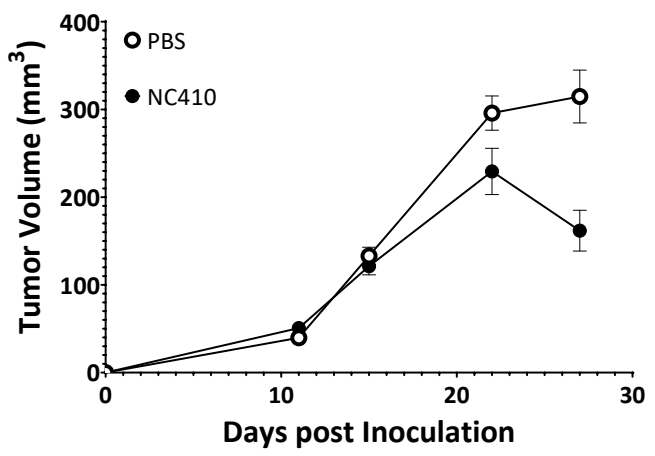

\begin{tabular}{|c|c|}
\hline Name & Description \\
\hline rec1M & $\begin{array}{c}\text { Neo-epitope of MMP-2,9,13 mediated degradation } \\
\text { of type I collagen }\end{array}$ \\
\hline C3M & Type III collagen degradation by MMP \\
\hline C4M & Type IV collagen degradation by MMP \\
\hline C6M & $\begin{array}{l}\text { Neo-epitope of MMP-2 mediated degradation of } \\
\text { type VI collagen }\end{array}$ \\
\hline PRO-C3 & $\begin{array}{l}\text { Pro-peptide of type III collagen / ECM formation / } \\
\text { fibroblast activity }\end{array}$ \\
\hline PRO-C6 & Pro-peptide of type VI collagen \\
\hline PRO-C23 & C-terminal of type XXIII collagen \\
\hline VICM & $\begin{array}{c}\text { Neo-epitope of MMP-2,8, trypsin mediated } \\
\text { degradation of citrullinated vimentin }\end{array}$ \\
\hline C4GzB & $\begin{array}{c}\text { Type IV collagen degraded by Granzyme B (T-cell } \\
\text { activity/infiltration }\end{array}$ \\
\hline
\end{tabular}

D)

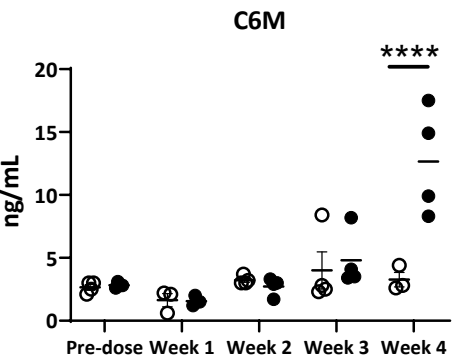

rec1M

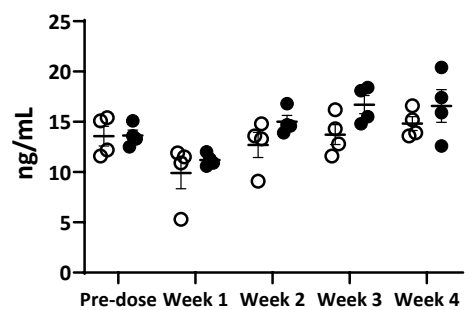

PRO-C6

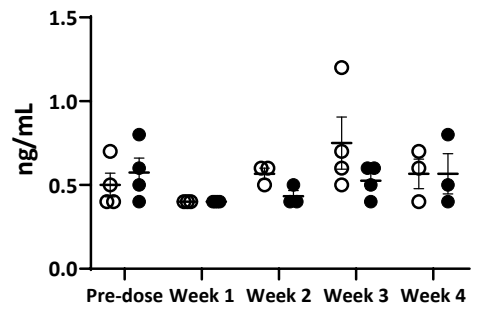

C4GzB

VICM

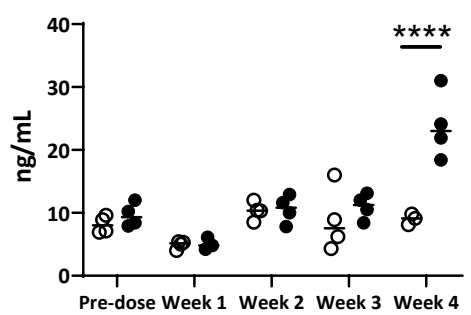

C3M

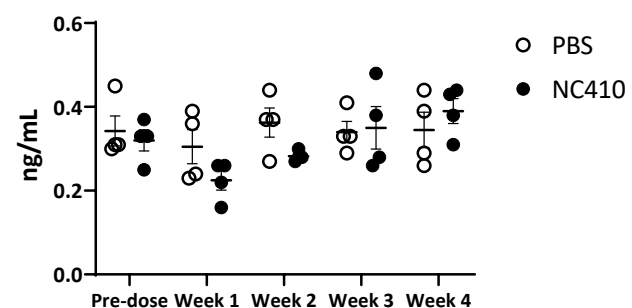

C4M

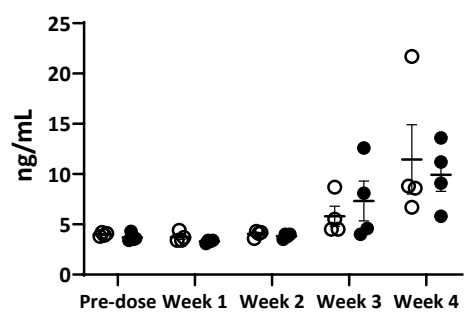

PRO-C23

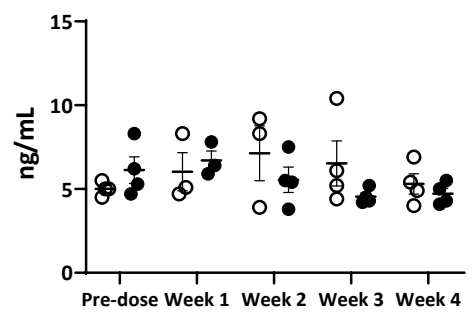

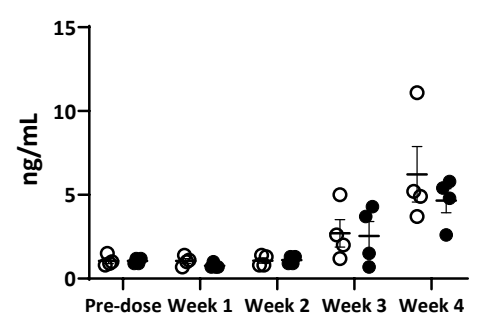

rPRO-C3

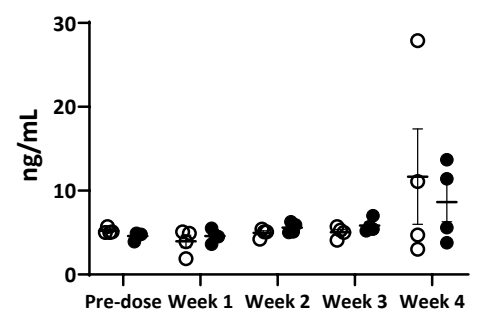


A)
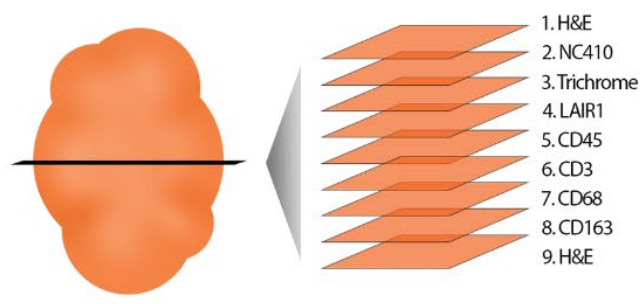

B)
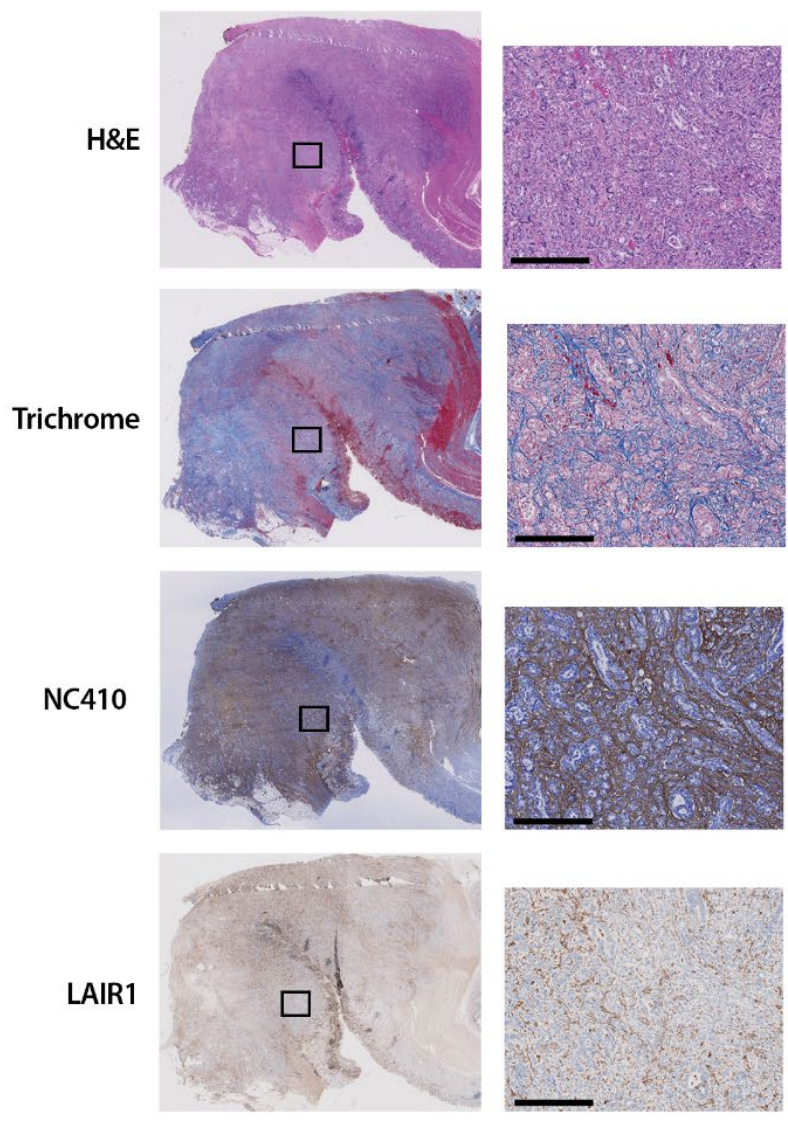

E)
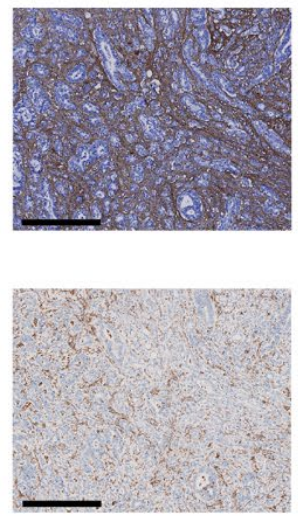

Glioblastoma
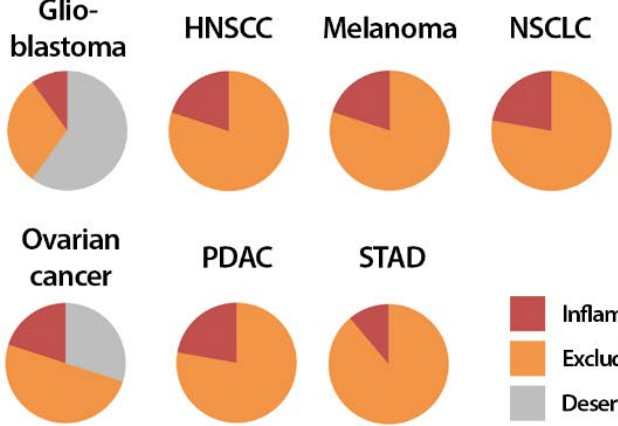

PDAC
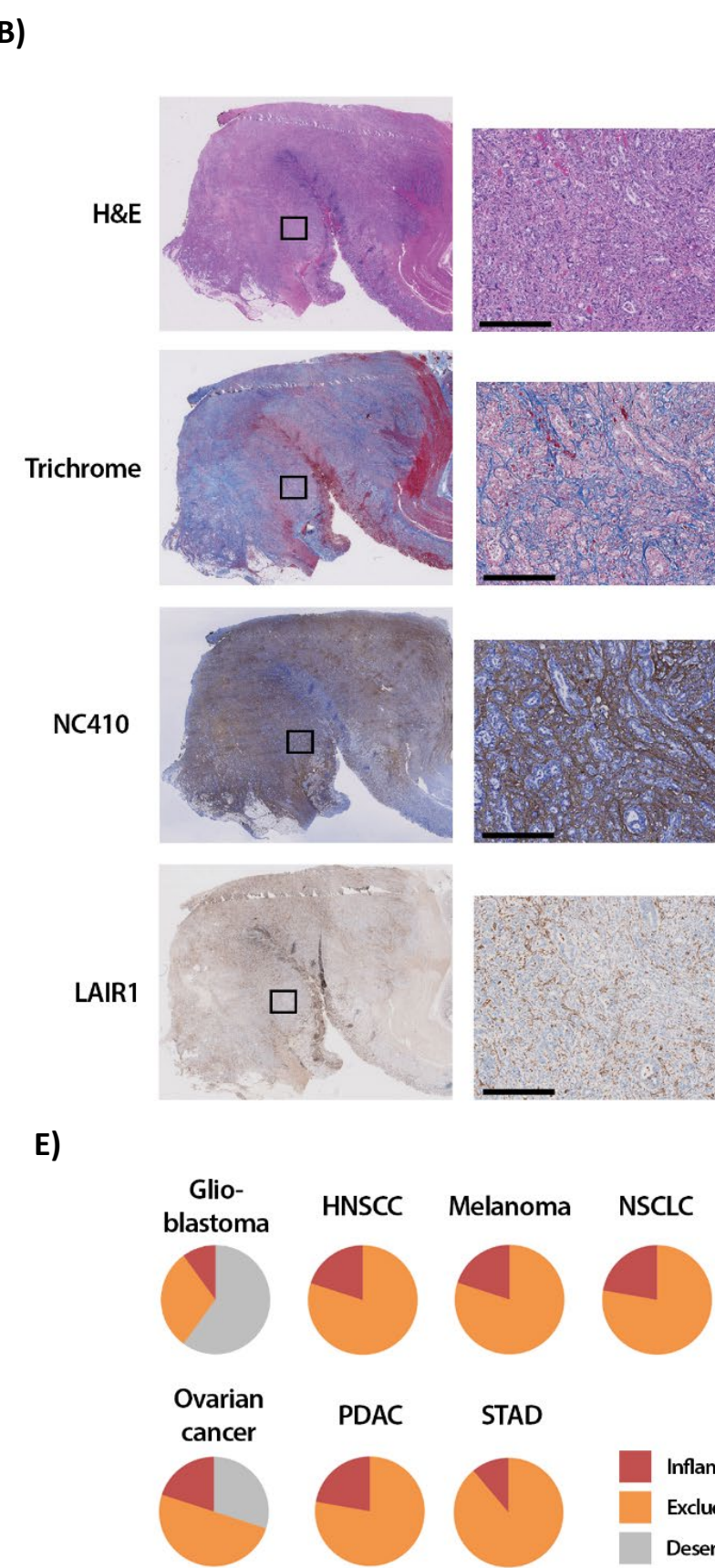

C)

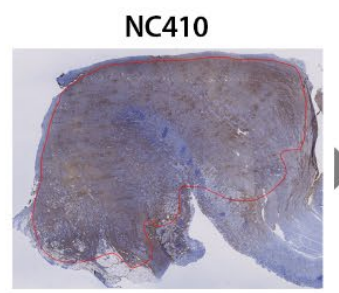

LAIR1
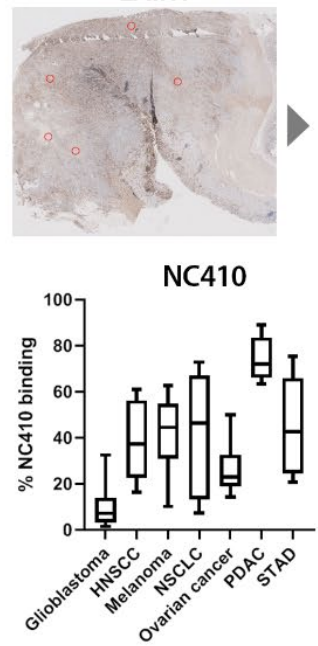

D)
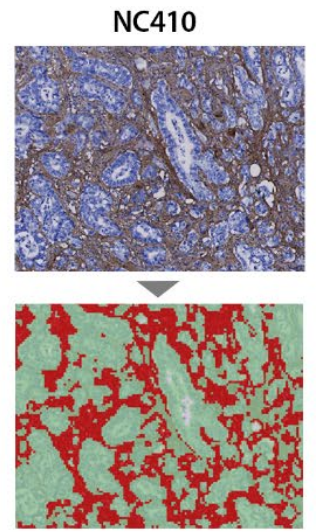

F)

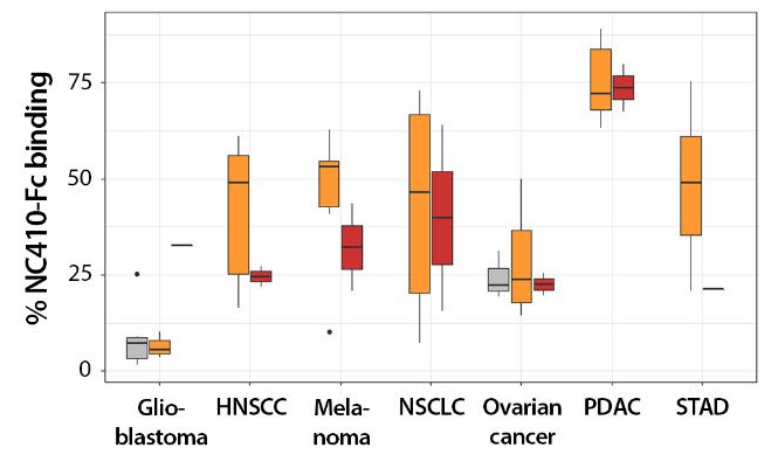 \\ Figure 6-NC410 binds to collagen rich tumors with an immune-excluded phenotype}




\section{Supplemental Figure 1- NC410 blocks LAIR-1 functional interaction with collagen.}

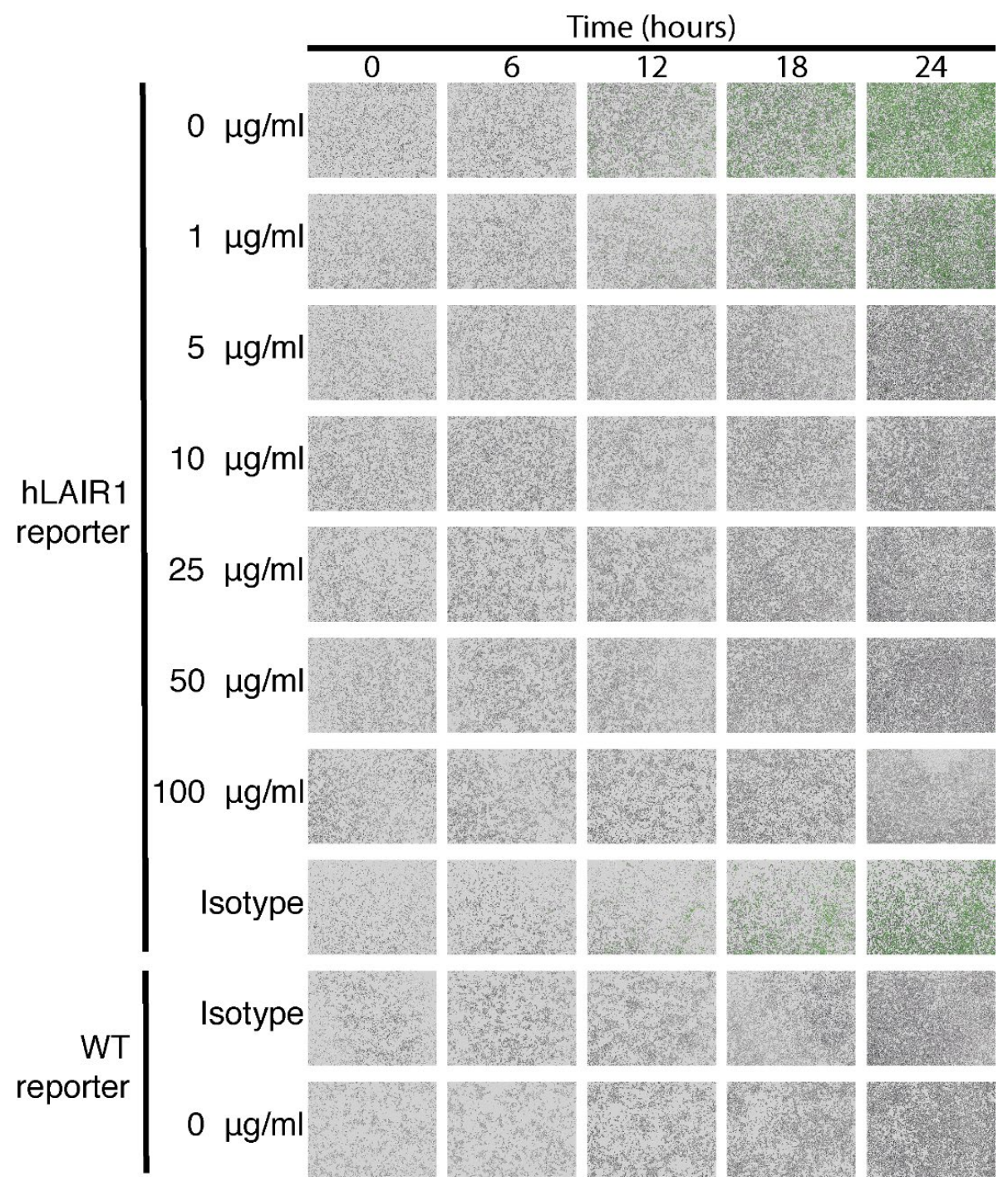

NC410 dose response measured by incucyte imaging during $24 \mathrm{~h}$. Representative microscopic images (10x) of WT and hLAIR-1 reporter cells over time. NC410 protein was added at increasing concentrations and inhibited collagen I ( $5 \mathrm{ug} / \mathrm{mL}$ ) mediated NFAT-GFP signaling through LAIR-1 binding. Isotype control was used at the highest concentration $(100 \mathrm{ug} / \mathrm{mL})$ and showed no inhibition of NFAT-GFP signaling. Pictures were taken every $1 \mathrm{~h}$ for $24 \mathrm{~h}$. Representative images from 1 out of 3 independently performed experiments (each with experimental triplicates). 


\section{Supplemental Figure 2- HT-29 mRNA collagen expression by RNA sequencing}

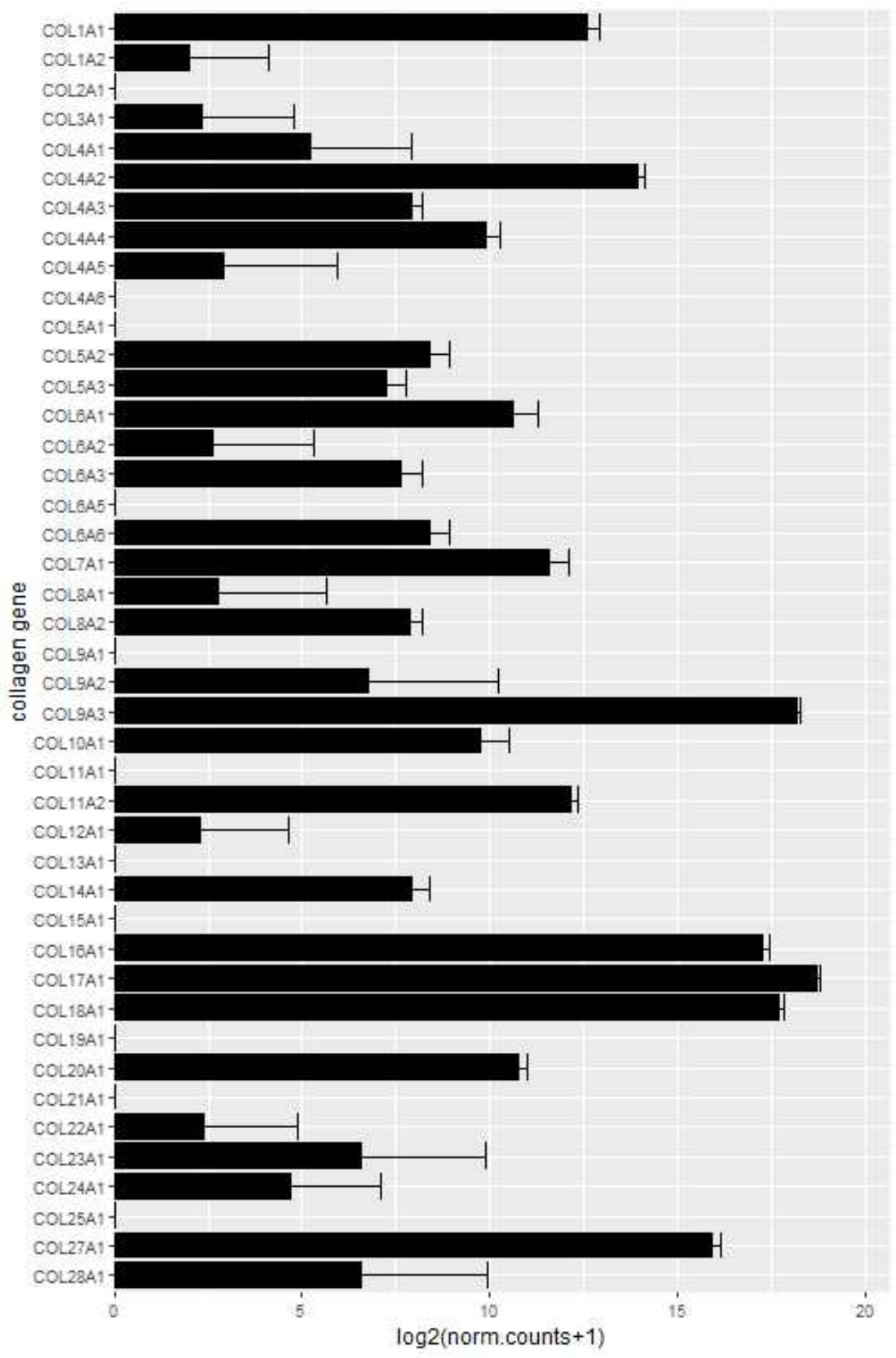

For collagen gene expression analysis of HT-29 cells, data was acquired from the public dataset GSE41586 (https://pubmed.ncbi.nlm.nih.gov/23902433/). Raw count data of untreated HT-29 cells was retrieved and normalized using the DESeq2 package (v1.28.1) in R (v4.0.2). Data was then log2 transformed and plotted using the ggplot package (v3.3.2). 


\section{Supplemental Figure 3- HT-29 collagen expression}

A)

B)

C)
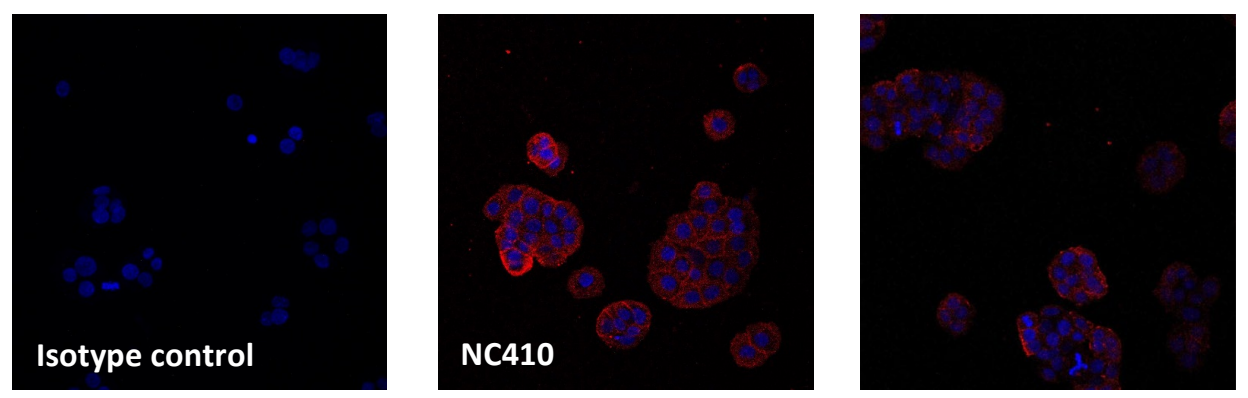

D)

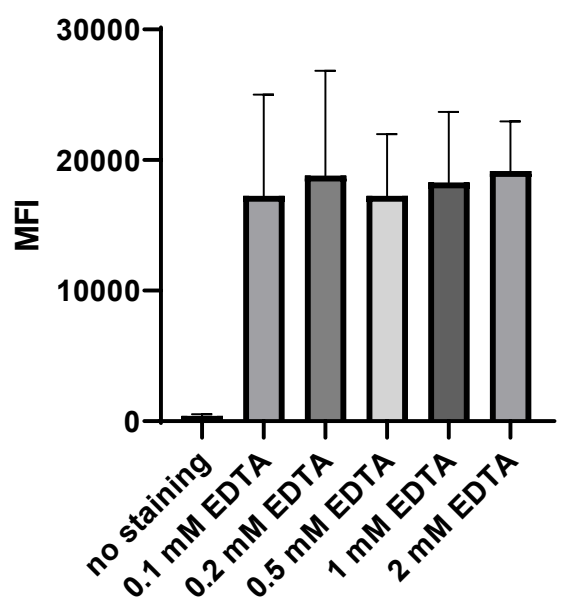

E)

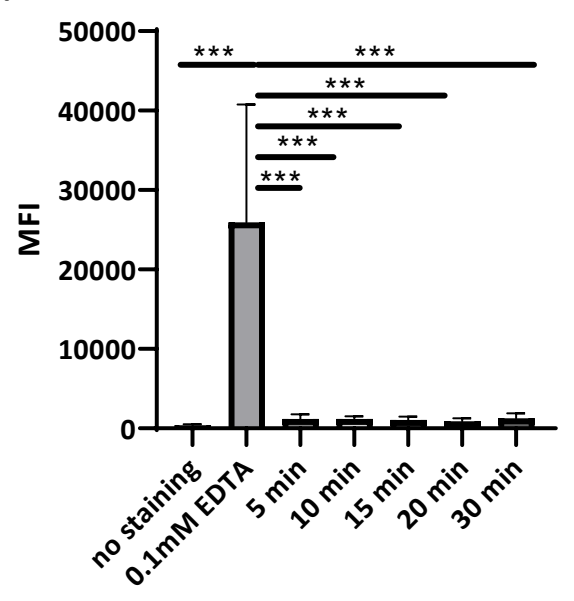

Immunofluorescence analysis of HT-29 cells stained with A) isotype control, B) NC410 or C) pan-collagen antibody. 40x magnification D) HT-29 cells were removed from culture flasks using increasing concentrations of EDTA for 10 min. MFI of NC410 staining is shown, demonstrating that EDTA treated HT29 cells keep surface collagen expression. E) HT-29 cells treated with $0.1 \mathrm{mM}$ collagenase lose NC410 binding. Data from 3 independently performed experiments. 

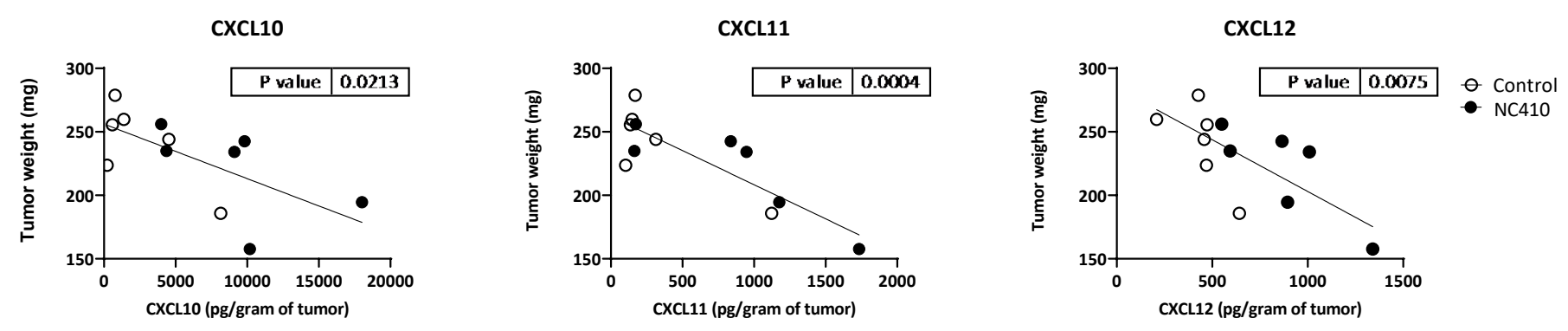

CXCL10, CXCL11 and CXCL12 chemokine expression correlate with reduced tumor growth at day 27 after HT-29 injection. P value was calculated by linear regression with F test. 6 mice per group were used. Black circles indicate NC410 treatment and open circles indicates control treatment. 


\section{Tumor tissue}
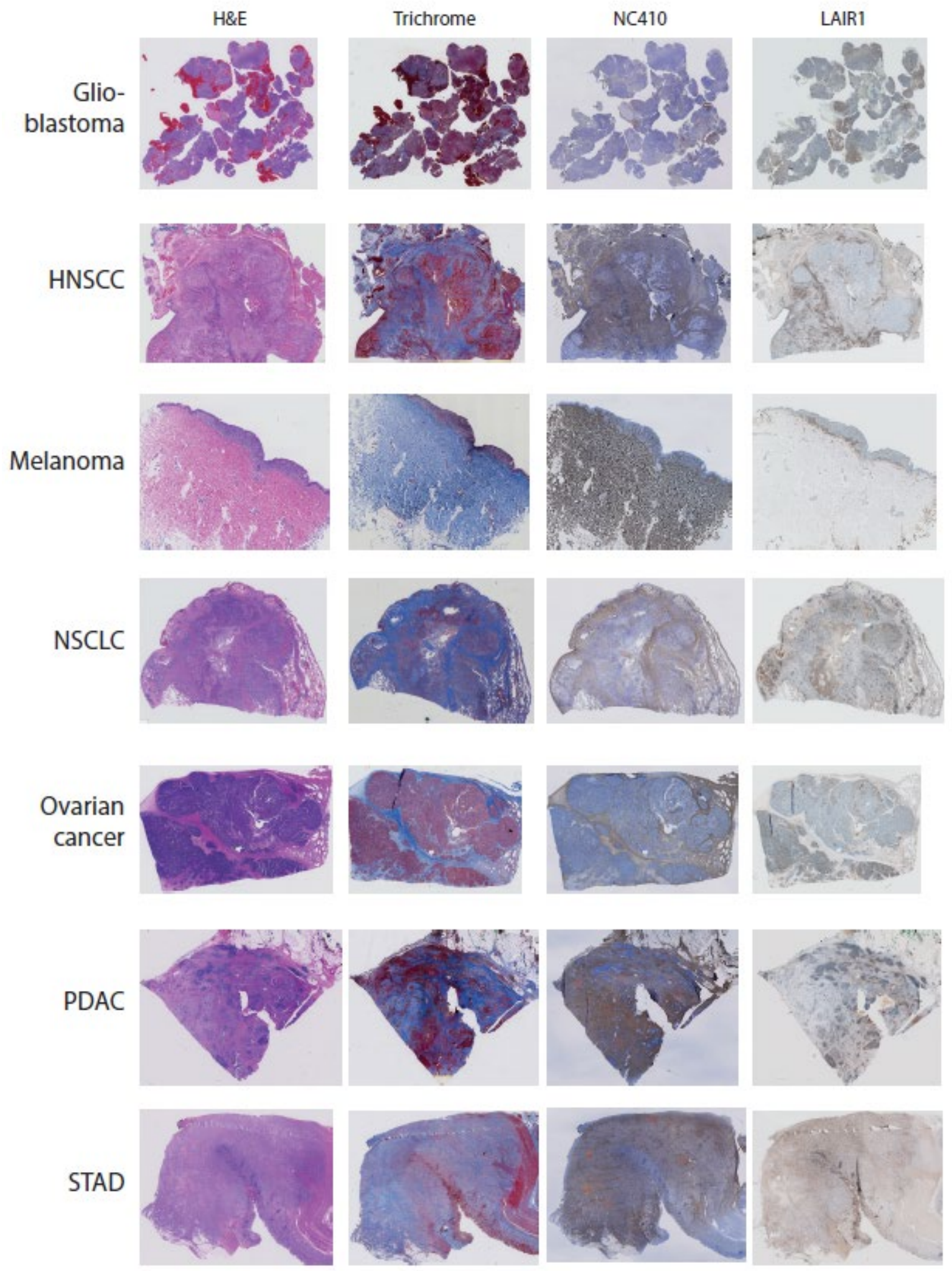

Representative hematoxylin and eosin (H\&E), Masson Trichrome, NC410 and LAIR-1 staining for 7 different tumor types (Head and neck (HNSC), melanoma, -non-small cell lung carcinoma (NSCLC), ovarian, pancreatic (PDAC) and stomach cancer (STAD)) 


\section{Supplemental Figure 6- Immunohistochemical analysis of healthy tissue}

\section{Healthy tissue}
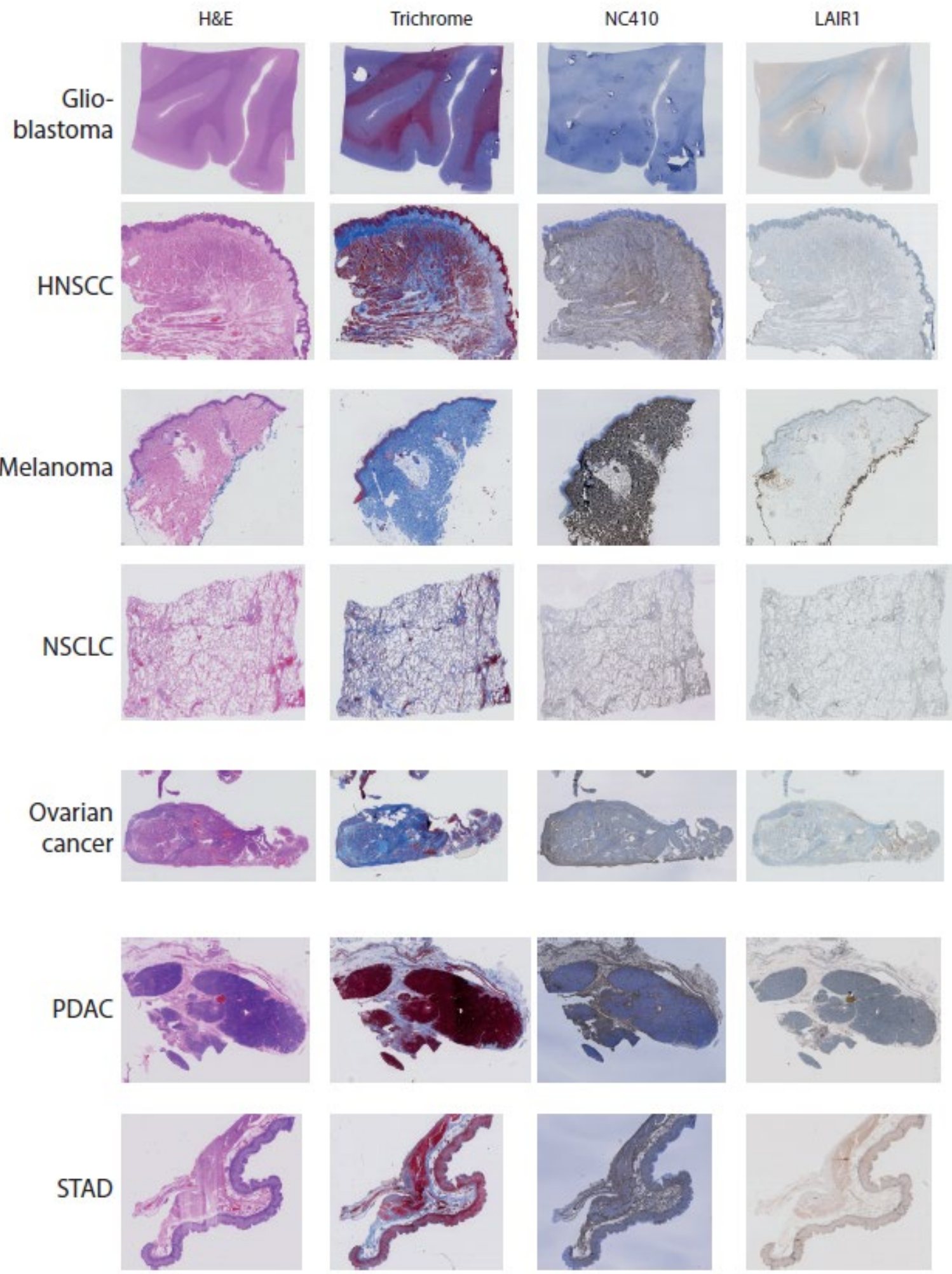

Representative hematoxylin and eosin (H\&E), Masson Trichrome, NC410 and LAIR-1 staining for healthy tissue matching the tumors used in Supplemental Figure 5. 
A)
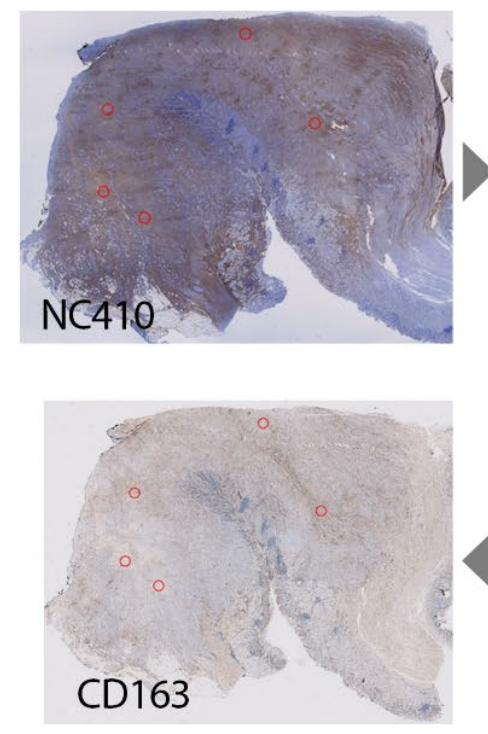
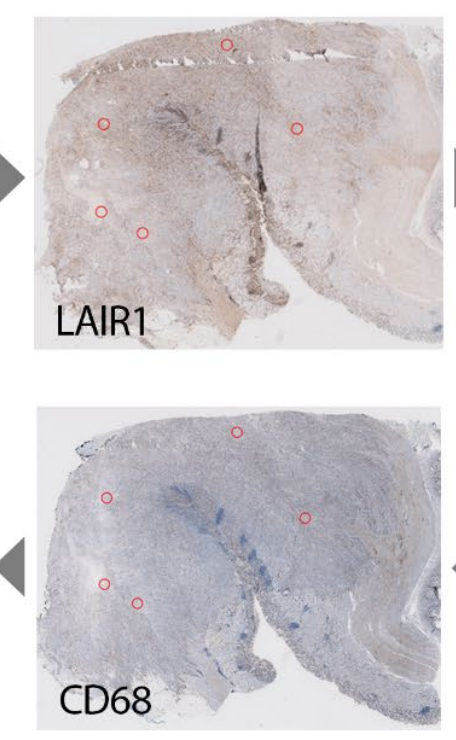

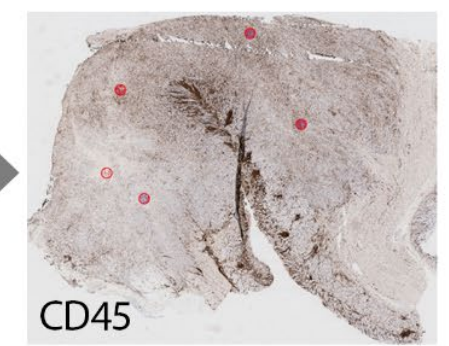

B)

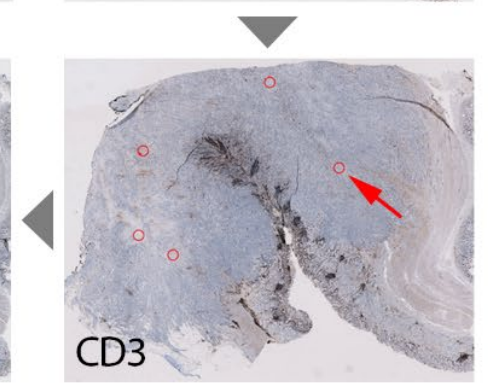

C)

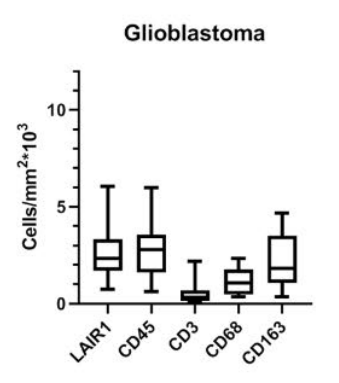

NSCLC

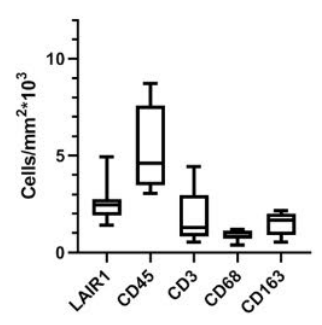

HNSCC

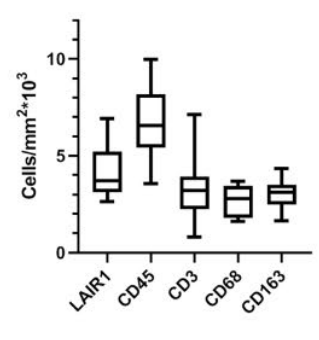

Ovarian cancer

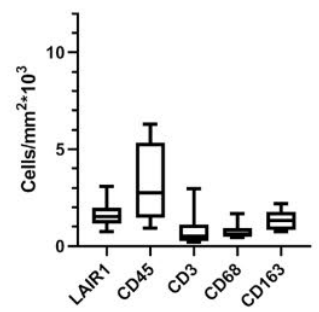

Melanoma

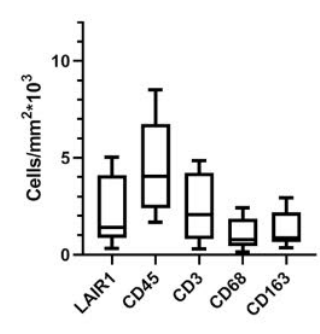

PDAC
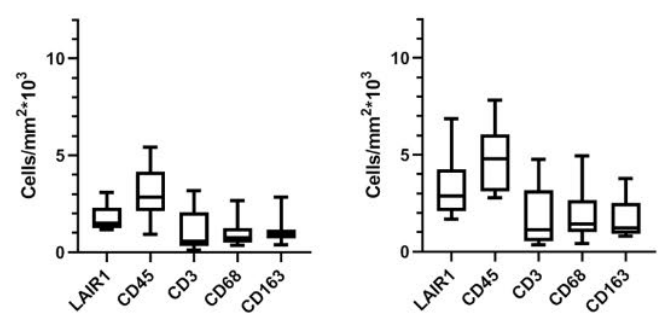

A) Representative LAIR-1 FC, NC410, CD45, CD3, CD68 and CD163 staining in a stomach tumor specimen. B) For quantification of the immune cell counts, regions of interest (ROIs) were annotated on the tissue slides by drawing circles with a diameter of $600 \mu \mathrm{m}$ at five random spots within the NC410 positive part of the tumor. Positive cells were quantified using the Positive Cell Detection tool. C) Number of positive LAIR-1 FC, CD45, CD3, CD68 and CD163 cells are shown across 7 different tumor types (Head and neck (HNSC), melanoma, non-small cell lung carcinoma (NSCLC), ovarian, pancreatic (PDAC) and stomach cancer $(\mathrm{STAD}))$. 


\section{Supplemental Figure 8- Tumor immune phenotyping}
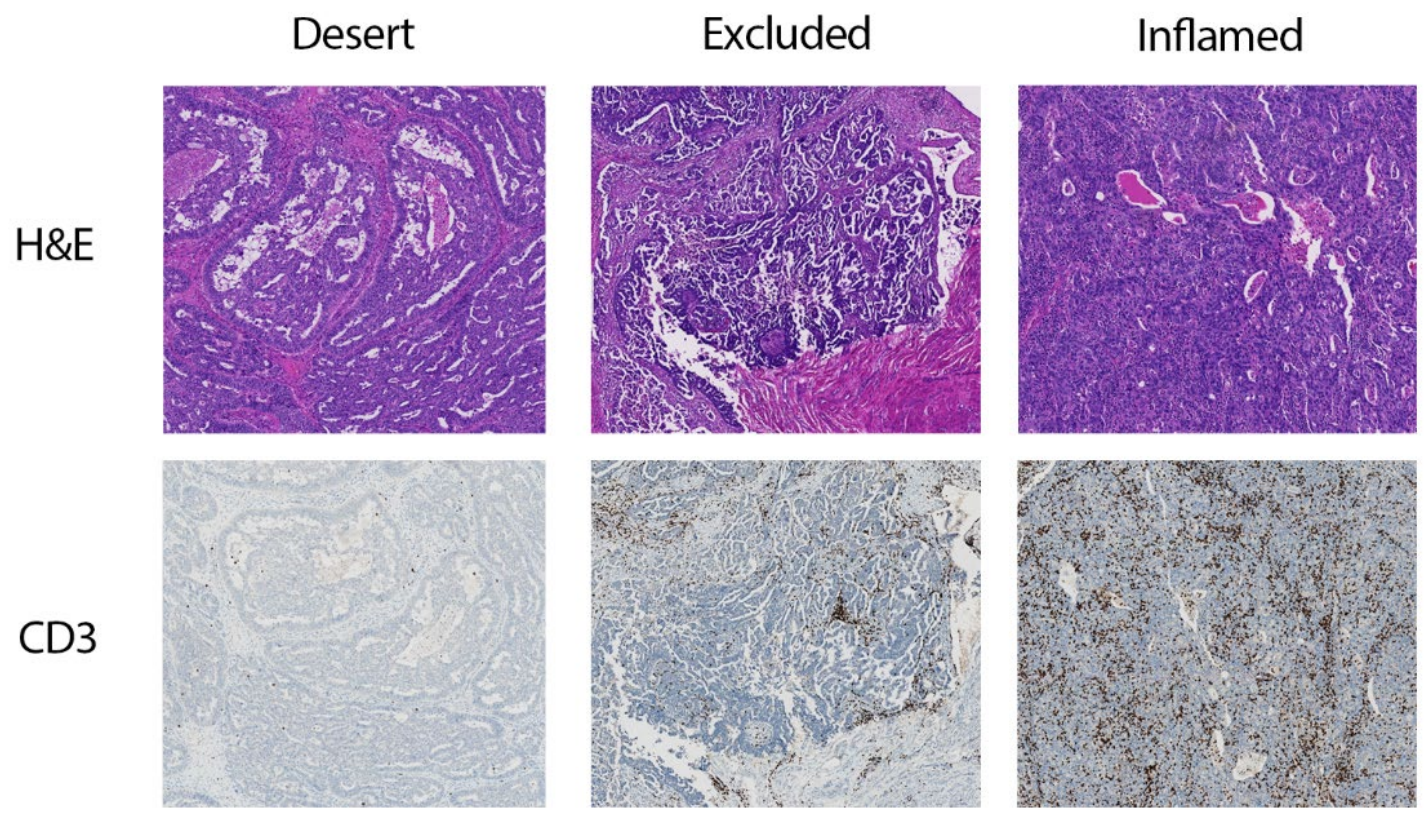

Representative image of the 3 different immune phenotypes, immune desert, immune excluded and immune inflamed in an ovarian tumor specimen. 